Daniele Angella*, Tatsuo Suwa, Nicoletta Tardini, and Adriano Tomassini

\title{
Note on Dolbeault cohomology and Hodge structures up to bimeromorphisms
}

https://doi.org/10.1515/coma-2020-0103

Received July 16, 2020; accepted August 17, 2020

Abstract: We construct a simply-connected compact complex non-Kähler manifold satisfying the $\partial \bar{\partial}$-Lemma, and endowed with a balanced metric. To this aim, we were initially aimed at investigating the stability of the property of satisfying the $\partial \bar{\partial}$-Lemma under modifications of compact complex manifolds and orbifolds. This question has been recently addressed and answered in $[34,39,40,50]$ with different techniques. Here, we provide a different approach using Čech cohomology theory to study the Dolbeault cohomology of the blowup $\tilde{X}_{Z}$ of a compact complex manifold $X$ along a submanifold $Z$ admitting a holomorphically contractible neighbourhood.

Keywords: complex manifold, non-Kähler geometry, $\partial \bar{\partial}$-Lemma, Hodge decomposition, modification, blowup, Dolbeault cohomology, orbifold

MSC: 32Q99, 32C35, $32 \mathrm{~S} 45$

\section{Introduction}

The $\partial \bar{\partial}$-Lemma is a strong cohomological decomposition property defined for complex manifolds, which is satisfied for example by algebraic projective manifolds and, more generally, by compact Kähler manifolds. The property is closely related to the fact that the Dolbeault cohomology provides a Hodge structure on the de Rham cohomology (cf. Subsection 1.5 below).

This property yields also strong topological obstructions: the real homotopy type of a compact complex manifold satisfying the $\partial \bar{\partial}$-Lemma is a formal consequence of its cohomology ring [16]. Complex non-Kähler manifolds usually do not satisfy the $\partial \bar{\partial}$-Lemma: for example, it is never satisfied by compact non-tori nilmanifolds [23]. On the other hand, some examples of compact complex non-Kähler manifolds satisfying the $\partial \bar{\partial}$ Lemma are provided by Moišhezon manifolds and manifolds in class $\mathcal{C}$ of Fujiki thanks to [16, Theorem 5.22], see [24] for a concrete example. By the results contained in [13, Corollary 3.13], [28, Theorem 1] and thanks to the stability property of the $\partial \bar{\partial}$-Lemma for small deformations [47, Proposition 9.21], [52, Theorem 5.12] one can produce examples of compact complex manifolds satisfying the $\partial \bar{\partial}$-Lemma and not bimeromorphic to Kähler manifolds. Other examples of this kind can be found among solvmanifolds [4, 5, 26]; moreover other examples are provided by Clemens manifolds $[18,19]$, which are constructed by combining modifications and deformations.

\footnotetext{
`Corresponding Author: Daniele Angella: Dipartimento di Matematica e Informatica “Ulisse Dini”, Università degli Studi di Firenze, viale Morgagni 67/a, 50134 Firenze, Italy, E-mail: daniele.angella@gmail.com, daniele.angella@unifi.it

Tatsuo Suwa: Department of Mathematics, Hokkaido University, Sapporo 060-0810, Japan, E-mail: tsuwa@sci.hokudai.ac.jp Nicoletta Tardini: Dipartimento di Matematica e Informatica “Ulisse Dini”, Università degli Studi di Firenze, viale Morgagni 67/a, 50134 Firenze, Italy, E-mail: nicoletta.tardini@gmail.com

Current address: Dipartimento di Scienze Matematiche, Fisiche e Informatiche, Unità di Matematica e Informatica, Università degli Studi di Parma, Parco Area delle Scienze 53/A, 43124 Parma, Italy

Adriano Tomassini: Dipartimento di Scienze Matematiche, Fisiche e Informatiche, Unità di Matematica e Informatica, Università degli Studi di Parma, Parco Area delle Scienze 53/A, 43124 Parma, Italy, E-mail: adriano.tomassini@unipr.it
} 
The main aim of this note is to construct a simply-connected compact complex non-Kähler manifold satisfying the $\partial \bar{\partial}$-Lemma.

The theorem in [16, Theorem 5.22] states that, for a modification $\tilde{X} \rightarrow X$ of compact complex manifolds, the property of $\partial \bar{\partial}$-Lemma is preserved from $\tilde{X}$ to $X$. So, it is natural to ask whether it is in fact an invariant property by modifications. This is true, for example, for compact complex surfaces, thanks to the topological Lamari's and Buchdahl's criterion [12, 27]. Note that, in higher dimension, the Kähler property is not stable under modifications; but there are weaker metric properties that are, for example the balanced condition in the sense of Michelsohn [3, Corollary 5.7] or the strongly-Gauduchon condition in the sense of Popovici [31, Theorem 1.3]. In fact, it is conjectured that the metric balanced condition and the cohomological $\partial \bar{\partial}$-Lemma property are strictly related to each other, see for example [33, Conjecture 6.1], see also [32, 46]; and this provides another motivation for the above question.

In this note, we deal with the Dolbeault cohomology of the blow-up along submanifolds. The strategy we follow is sheaf-theoretic, more precisely Čech-cohomological, in the spirit of [43]. The de Rham case in the Kähler context is considered in [47, Theorem 7.31]. For our argument, we need to assume that the centre admits a holomorphically contractible neighbourhood (this is clearly satisfied when blowing-up at a point, see also the explicit computations in Example 21) and another technical assumption (11) concerning the kernel and images of certain morphisms. We can then deduce that:

Theorem 13. Let $X$ be a compact complex manifold and $Z$ a closed submanifold of $X$. If both $X$ and the centre $Z$ admits a Hodge structure (in the sense of Definition 4), then the same holds for the blow-up $\mathrm{Bl}_{Z} X$ of $X$ along $Z$, provided that $Z$ admits a holomorphically contractible neighbourhood and the technical assumption (11) holds.

Along the way we give explicit expressions for the de Rham and Dolbeault cohomologies of $\mathrm{Bl}_{Z} X$ (see Propositions 16 and 19).

Hopefully, a further study of the cohomological properties of submanifolds (see Question 22) and a deeper use of techniques as the MacPherson's deformation to the normal cone (see Question 23), along with the Weak Factorization Theorem for bimeromorphic maps in the complex-analytic category [2, Theorem 0.3.1], [49], may allow to use the above techniques to prove in full generality the stability of the $\partial \bar{\partial}$-Lemma under modifications, see Remark 24.

During the preparation of this work, several other attempts to solve the same problems appeared [34, 39, 50], using different techniques. In particular, the work by Jonas Stelzig [38] finally ties up the problem, as far as now:

Theorem 1 ([39, Theorem 8], [40, Corollary 25]). The $\partial \bar{\partial}$-Lemma property is a bimeromorphic invariant if and only if it is invariant by restriction.

Even if Stelzig's theorem is clearly stronger than our Theorem 13, we think that our argument may be interesting and useful in providing a broader point of view for understanding (Čech-)Dolbeault cohomology.

The second and main aim of this note is to construct new explicit examples of compact complex manifolds satisfying the $\partial \bar{\partial}$-Lemma: in particular, we provide a simply-connected example, see Example 26 . To this aim, we need to work with orbifolds in the sense of Satake [36], and their desingularizations. We take advantage of Stelzig's general results, see Theorem 25. The construction of Example 26 goes as follows, see e.g. [10, 17]: we start from a manifold isomorphic to the Iwasawa manifold, which does not satisfy the $\partial \bar{\partial}$-Lemma; then we quotient it by a finite group of automorphisms; and then we resolve its singularities. Finally, by Theorem 25 , we get simply-connected examples of complex manifolds satisfying the $\partial \bar{\partial}$-Lemma:

Theorem 27. There exist a simply-connected compact complex non-Kähler manifold, (not even in class $\mathcal{C}$ of Fujiki,) that satisfy the $\partial \bar{\partial}$-Lemma. Our example admits a balanced metric.

As far as we know, these are the first explicit examples of simply-connected compact complex non-Kähler manifolds satisfying the $\partial \bar{\partial}$-Lemma in the literature. 


\section{Preliminaries on Čech-Dolbeault cohomology and $\partial \bar{\partial}$-Lemma}

In this Section, we recall the main definitions and results about relative Čech-de Rham and Čech-Dolbeault cohomologies; for more details and applications we refer to [41] and [43]. We also recall the $\partial \bar{\partial}$-Lemma and some of its characterizations.

\section{1 Čech-de Rham cohomology and relative de Rham cohomology}

Let $X$ be a smooth manifold. Let $\mathcal{U}=\left\{U_{0}, U_{1}\right\}$ be an open covering of $X$ and set $U_{01}:=U_{0} \cap U_{1}$. Denoting by $A^{h}(U)$ the space of (C-valued) smooth $h$-forms on an open set $U$ in $X$, we set

$$
A^{h}(\mathcal{U}):=A^{h}\left(U_{0}\right) \oplus A^{h}\left(U_{1}\right) \oplus A^{h-1}\left(U_{01}\right) .
$$

The differential operator $D: A^{h}(\mathcal{U}) \rightarrow A^{h+1}(\mathcal{U})$ defined by $D\left(\sigma_{0}, \sigma_{1}, \sigma_{01}\right)=\left(d \sigma_{0}, d \sigma_{1}, \sigma_{1}-\sigma_{0}-d \sigma_{01}\right)$ yields a differential complex $\left(A^{\bullet}(\mathcal{U}), D\right)$ : the Čech-de Rham cohomology associated to the covering $U$ is then defined by $H_{D}^{\bullet}(\mathcal{U})=\operatorname{ker} D / \operatorname{im} D$. The morphism $A^{h}(X) \rightarrow A^{h}(\mathcal{U})$ given by $\omega \mapsto\left(\left.\omega\right|_{U_{0}},\left.\omega\right|_{U_{1}}, 0\right)$ induces an isomorphism in cohomology, [41, Theorem 3.3],

$$
H_{d R}^{\bullet}(X) \stackrel{\sim}{\longrightarrow} H_{D}^{\bullet}(\mathcal{U}),
$$

whose inverse is given by assigning to the class of $\sigma=\left(\sigma_{0}, \sigma_{1}, \sigma_{01}\right)$ the class of the global $d$-closed form $\rho_{0} \sigma_{0}+\rho_{1} \sigma_{1}-d \rho_{0} \wedge \sigma_{01}$, where $\left(\rho_{0}, \rho_{1}\right)$ is a partition of unity subordinate to $U$. In the above $H_{d R}^{\cdot}(X)$ denotes the de Rham cohomology of $X$. The de Rham theorem says it is isomorphic to $H^{\bullet}(X ; \mathbb{C})$, the simplicial, singular or sheaf cohomology of $X$ with coefficents in $\mathbb{C}$. See [41] for further results, including cup product, integration on top-degree cohomology, duality.

Given a closed set $S$ in $X$, we can take $U_{0}:=X \backslash S$ and $U_{1}$ an open neighbourhood of $S$ in $X$, and the open covering $\mathcal{U}=\left\{U_{0}, U_{1}\right\}$. In this case, define $A^{p}\left(\mathcal{U}, U_{0}\right):=\left\{\sigma \in A^{h}(\mathcal{U}) \mid \sigma_{0}=0\right\}=A^{h}\left(U_{1}\right) \oplus A^{h-1}\left(U_{01}\right)$. Then $\left(A^{\bullet}\left(\mathcal{U}, U_{0}\right), D\right)$ is a differential sub-complex of $\left(A^{\bullet}(\mathcal{U}), D\right)$. Let $H_{D}^{h}\left(\mathcal{U}, U_{0}\right)$ denote the associated cohomology. From the short exact sequence

$$
0 \longrightarrow A^{\bullet}\left(\mathcal{U}, U_{0}\right) \longrightarrow A^{\bullet}(\mathcal{U}) \longrightarrow A^{\bullet}\left(U_{0}\right) \longrightarrow 0,
$$

where the first map is the inclusion and the second map is the projection on the first element, we obtain a long exact sequence in cohomology

$$
\cdots \longrightarrow H_{d R}^{h-1}\left(U_{0}\right) \stackrel{\delta}{\longrightarrow} H_{D}^{h}\left(\mathcal{U}, U_{0}\right) \stackrel{j^{*}}{\longrightarrow} H_{D}^{h}(\mathcal{U}) \stackrel{i^{*}}{\longrightarrow} H_{d R}^{h}\left(U_{0}\right) \longrightarrow \cdots .
$$

From this we see that $H_{D}^{h}\left(\mathcal{U}, U_{0}\right)$ is determined uniquely modulo canonical isomorphisms, independently of the choice of $U_{1}$. We denote it also by $H_{D}^{h}(X, X \backslash S)$ and call it the relative Čech-de Rham cohomology. We recall that excision holds: for any neighbourhood $U$ of $S$ in $X$, it holds $H_{D}^{h}(X, X \backslash S) \simeq H_{D}^{h}(U, U \backslash S)$. In fact we have, [42],

$$
H_{D}^{h}(X, X \backslash S) \simeq H^{h}(X, X \backslash S ; \mathbb{C}),
$$

the relative cohomology of the pair $(X, X \backslash S)$.

Consider now a smooth complex vector bundle $\pi: E \rightarrow M$ of rank $k$ on a smooth manifold $M$. Consider the bundle $\varpi: \pi^{\star} E \rightarrow E$ defined by the fibre product

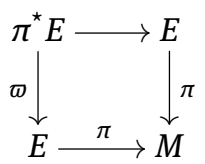

and its diagonal section $s_{\Delta}$. The zero-set of $s_{\Delta}$ is the image of the zero-section of $E$, which is identified with $M$. In this situation, the Thom class $\Psi_{E} \in H_{D}^{2 k}(E, E \backslash M)$ of $E$ is given as the localization of the top Chern class 
$c^{k}\left(\pi^{\star} E\right)$ by $s_{\Delta}$. That is: consider the covering $\mathcal{W}=\left\{W_{0}:=E \backslash M, W_{1}\right\}$ of $E$, where $W_{1}$ is a neighbourhood of $M$ in $E$; consider $\nabla_{0}$ a connection on $W_{0}$ such that $\nabla_{0} s_{\Delta}=0$, and $\nabla_{1}$ a connection on $W_{1}$; then the Chern class $c^{k}\left(\pi^{\star} E\right)$ is represented by $\left(c^{k}\left(\nabla_{0}\right), c^{k}\left(\nabla_{1}\right), c^{k}\left(\nabla_{0}, \nabla_{1}\right)\right) \in H_{D}^{2 k}(\mathcal{W}) \simeq H_{d R}^{2 k}(E)$, where $c^{k}\left(\nabla_{0}, \nabla_{1}\right)$ is the Bott difference form of $\nabla_{0}$ and $\nabla_{1}$; in fact, since $c^{k}\left(\nabla_{0}\right)=0$, this defines a class $\Psi_{E} \in H_{D}^{2 k}(E, E \backslash M)$ represented by $\left(\psi_{1}, \psi_{01}\right):=\left(c^{k}\left(\nabla_{1}\right), c^{k}\left(\nabla_{0}, \nabla_{1}\right)\right)$. It turns out that the map

$$
T_{E}: H_{d R}^{\bullet-2 k}(M) \stackrel{\sim}{\longrightarrow} H_{D}^{\bullet}(E, E \backslash M), \quad[\theta] \mapsto \Psi_{E} \smile \pi^{\star}[\theta]
$$

is an isomorphisms, [41, Theorem 5.3], called the Thom isomorphism, where the cup product $\Psi_{E} \smile \pi^{\star}[\theta]$ is represented by $\left(\psi_{1} \wedge \pi^{\star} \theta, \psi_{01} \wedge \pi^{*} \theta\right)$. Its inverse is the integration along the fibres:

$$
\pi_{\star}: H_{D}^{\bullet}(E, E \backslash M) \longrightarrow H_{d R}^{\bullet-2 k}(M), \quad \pi_{\star}\left(\sigma_{1}, \sigma_{01}\right)=\left(\pi_{1}\right)_{\star} \sigma_{1}+\left(\pi_{01}\right) \star \sigma_{01},
$$

where $\pi_{1}$ is the restriction of $\pi$ to a bundle $T_{1}$ of disks of complex dimension $k$ in $W_{1}$, and $\pi_{01}$ is the restriction of $\pi$ to the bundle $T_{01}=-\partial T_{1}$ of spheres of real dimension $2 k-1$ with opposite orientation. In particular, the Thom class $\Psi_{E}$ is characterized in $H_{D}^{2 k}(E, E \backslash M)$ by the property $\pi_{\star} \Psi_{E}=1$. Finally, we recall the projection formula, [41, Ch.II, Proposition 5.1]: for $\sigma \in A^{p}\left(\mathcal{W}, W_{0}\right), \theta \in A^{q}(M)$,

$$
\pi \star\left(\sigma \smile \pi^{\star} \theta\right)=\pi \star \sigma \wedge \theta .
$$

Given a closed complex submanifold $Z$, of complex codimension $k$, of a complex manifold $X$, of complex dimension $n$, we can define the Thom isomorphism and the Thom class of $Z$ as follows. Consider the normal bundle $\pi: N_{Z \mid X} \rightarrow Z$, of complex rank $k$. By the Tubular Neighbourhood Theorem, there exist neighbourhoods $U$ of $Z$ in $X$, and $W$ of $Z$ as zero section in $N_{Z \mid X}$, and a smooth diffeomorphism $\varphi: U \rightarrow W$ such that $\left.\varphi\right|_{Z}=$ id. Then, setting $N=N_{Z \mid X}$, we get isomorphisms

$$
H_{D}^{\bullet}(X, X \backslash Z) \simeq H_{D}^{\bullet}(U, U \backslash Z) \underset{\varphi^{*}}{\sim} H_{D}^{\bullet}(W, W \backslash Z) \simeq H_{D}^{\bullet}(N, N \backslash Z)
$$

Define the Thom class $\Psi_{Z} \in H_{D}^{2 k}(X, X \backslash Z)$ of $Z$ as the image of $\Psi_{N_{Z \mid X}}$ via the above isomorphisms, and the Thom isomorphism $T_{Z}: H_{D}^{\cdot-2 k}(Z) \stackrel{\sim}{\rightarrow} H_{D}^{*}(X, X \backslash Z)$ as $T_{Z}(z)=\Psi_{Z} \smile r^{\star} z$, where $r=\pi \circ \varphi: U \rightarrow Z$.

\section{2 Čech-Dolbeault cohomology}

Let $X$ be a complex manifold and let $A^{p, q}(U)$ be the space of smooth $(p, q)$-forms on an open set $U$ in $X$. Let $\mathcal{U}=\left\{U_{0}, U_{1}\right\}$ be an open covering of $X$ and consider

$$
A^{p, q}(\mathcal{U}):=A^{p, q}\left(U_{0}\right) \oplus A^{p, q}\left(U_{1}\right) \oplus A^{p, q-1}\left(U_{01}\right) .
$$

The differential operator $\bar{D}: A^{p, q}(\mathcal{U}) \rightarrow A^{p, q+1}(\mathcal{U})$ is defined on every element $\left(\xi_{0}, \xi_{1}, \xi_{01}\right) \in A^{p, q}(\mathcal{U})$ by

$$
\bar{D}\left(\xi_{0}, \xi_{1}, \xi_{01}\right)=\left(\bar{\partial} \xi_{0}, \bar{\partial} \xi_{1}, \xi_{1}-\xi_{0}-\bar{\partial} \xi_{01}\right) \text {. }
$$

The Čech-Dolbeault cohomology associated to the covering $U$ is then defined by $H_{\bar{D}}^{\bullet, \bullet}(\mathcal{U})=\operatorname{ker} \bar{D} / \operatorname{im} \bar{D}$ (see [43] where this definition is given for an arbitrary open covering of the manifold $X)$. The morphism $A^{p, q}(X) \rightarrow$ $A^{p, q}(\mathcal{U})$ given by $\omega \mapsto\left(\left.\left.\omega\right|_{U_{0}} \omega\right|_{U_{1}}, 0\right)$ induces an isomorphism in cohomology

$$
H_{\bar{\partial}}^{\bullet \bullet \bullet}(X) \stackrel{\sim}{\longrightarrow} H_{\bar{D}}^{\bullet \bullet \bullet}(\mathcal{U})
$$

where $H_{\vec{\partial}}^{\bullet \bullet \bullet}(X)$ denotes the Dolbeault cohomology of $X$, [43, Theorem 1.2]. In particular, the definition is independent of the choice of the covering of $X$. Moreover, the inverse map is given by assigning to the class of $\xi=\left(\xi_{0}, \xi_{1}, \xi_{01}\right)$ the class of the global $\bar{\partial}$-closed form $\rho_{0} \xi_{0}+\rho_{1} \xi_{1}-\bar{\partial} \rho_{0} \wedge \xi_{01}$, where $\left(\rho_{0}, \rho_{1}\right)$ is a partition of unity subordinate to $\mathcal{U}$.

One can define cup product, integration on top-degree cohomology and Kodaira-Serre duality and they turn out to be compatible with the above isomorphism (cf. [43] for more details). 


\subsection{Relative Čech-Dolbeault cohomology}

Let $S$ be a closed set in $X$. We set $U_{0}=X \backslash S$ and $U_{1}$ to be an open neighbourhood of $S$ in $X$, and we consider the associated covering $\mathcal{U}=\left\{U_{0}, U_{1}\right\}$ of $X$. For any $p, q$, we set

$$
A^{p, q}\left(\mathcal{U}, U_{0}\right):=\left\{\xi \in A^{p, q}(\mathcal{U}) \mid \xi_{0}=0\right\}=A^{p, q}\left(U_{1}\right) \oplus A^{p, q-1}\left(U_{01}\right) .
$$

Then $\left(A^{p, \bullet}\left(\mathcal{U}, U_{0}\right), \bar{D}\right)$ is a subcomplex of $\left(A^{p, \bullet}(\mathcal{U}), \bar{D}\right)$. Let $H_{\bar{D}}^{p, q}\left(\mathcal{U}, U_{0}\right)$ be the cohomology associated to $\left(A^{p, \bullet}\left(\mathcal{U}, U_{0}\right), \bar{D}\right)$. From the short exact sequence

$$
0 \longrightarrow A^{p, \bullet}\left(\mathcal{U}, U_{0}\right) \longrightarrow A^{p, \bullet}(\mathcal{U}) \longrightarrow A^{p, \bullet}\left(U_{0}\right) \longrightarrow 0,
$$

where the first map is the inclusion and the second map is the projection on the first element, we obtain a long exact sequence in cohomology

$$
\cdots \longrightarrow H_{\bar{\partial}}^{p, q-1}\left(U_{0}\right) \stackrel{\delta}{\longrightarrow} H_{\bar{D}}^{p, q}\left(\mathcal{U}, U_{0}\right) \stackrel{j^{*}}{\longrightarrow} H_{\bar{D}}^{p, q}(\mathcal{U}) \stackrel{i^{*}}{\longrightarrow} H_{\bar{\partial}}^{p, q}\left(U_{0}\right) \longrightarrow \cdots .
$$

Therefore, $H_{\bar{D}}^{\bullet \bullet \bullet}\left(\mathcal{U}, U_{0}\right)$ is determined uniquely modulo canonical isomorphism, independently of the choice of $U_{1}$. We denote it also by $H_{\bar{D}}^{\bullet \bullet \bullet}(X, X \backslash S)$ and we call it the relative Čech-Dolbeault cohomology of $X$, see [43, Section 2], where it is denoted by $H_{\bar{\partial}}^{\bullet \bullet}(X, X \backslash S)$. We recall that excision holds: for any neighbourhood $U$ of $S$ in $X$, it holds $H_{\bar{D}}^{\bullet \cdot \bullet}(X, X \backslash S) \simeq H_{\bar{D}}^{\bullet \bullet}(U, U \backslash S)$. In fact we have, [44],

$$
H_{\bar{D}}^{p, q}(X, X \backslash S) \simeq H^{q}\left(X, X \backslash S ; \Omega^{p}\right),
$$

the relative cohomology of the pair $(X, X \backslash S)$ with coefficients in the sheaf $\Omega^{p}$ of holomorphic $p$-forms.

Together with integration theory, the relative Čech-Dolbeault cohomology has been used to study the localization of characteristic classes, see $[1,43]$, and has found more recent applications to hyperfunction theory, see [25].

Notice that if $X$ and $\tilde{X}$ are complex manifolds, $S$ and $\tilde{S}$ are closed sets in $X$ and $\tilde{X}$ respectively and $f: \tilde{X} \rightarrow X$ is a holomorphic map such that $f(\tilde{S}) \subset S$ and $f(\tilde{X} \backslash \tilde{S}) \subset f(X \backslash S)$, then $f$ induces a natural map in relative cohomology. More precisely, let $U_{0}:=X \backslash S, \tilde{U}_{0}:=\tilde{X} \backslash \tilde{S}$ and let $U_{1}, \tilde{U}_{1}$ be open neighborhoods of $S$ and $\tilde{S}$ in $X$ and $\tilde{X}$ respectively, chosen in such a way that $f\left(\tilde{U}_{1}\right) \subset U_{1}$. Let $\mathcal{U}:=\left\{U_{0}, U_{1}\right\}$ and $\tilde{U}:=\left\{\tilde{U}_{0}, \tilde{U}_{1}\right\}$ be open coverings of $X$ and $\tilde{X}$ respectively, then we have a morphism

$$
f^{\star}: A^{\bullet \bullet \bullet}\left(\mathcal{U}, U_{0}\right) \longrightarrow A^{\bullet \cdot \bullet}\left(\tilde{\mathcal{U}}, \tilde{U}_{0}\right)
$$

defined on every element $\left(\xi_{1}, \xi_{01}\right) \in A^{\bullet \cdot \bullet}\left(\mathcal{U}, U_{0}\right)$ as

$$
f^{\star}\left(\xi_{1}, \xi_{01}\right):=\left(f^{\star} \xi_{1}, f^{\star} \xi_{01}\right)
$$

which induces a morphism in relative cohomology

$$
f^{\star}: H_{\bar{D}}^{\bullet \bullet \bullet}(X, X \backslash S) \longrightarrow H_{\bar{D}}^{\bullet \bullet \bullet}(\tilde{X}, \tilde{X} \backslash \tilde{S}) .
$$

\subsection{Dolbeault-Thom morphism}

We consider a holomorphic vector bundle $\pi: E \rightarrow X$ of rank $k$ on a complex manifold $X$ and we identify $X$ with the image of the zero section. In this situation we have the Dolbeault-Thom class, $\bar{\partial}$-Thom class for short, $\bar{\Psi}_{E} \in H_{\bar{D}}^{k, k}(E, E \backslash X)$ and the Dolbeault-Thom morphism, $\bar{\partial}$-Thom morphism for short, $\bar{T}_{E}: H_{\bar{\partial}}^{p-k, q-k}(X) \rightarrow$ $H_{\bar{D}}^{p, q}(E, E \backslash X)$.

They are given as follows, see $[1,43]$. Consider the fibre product

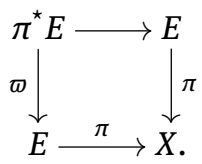


The bundle $\varpi: \pi^{\star} E \rightarrow E$ admits the diagonal section $s_{\Delta}$, whose zero set is $X \subset E$. The Dolbeault-Thom class $\bar{\Psi}_{E}$ is the localization of the top Atiyah class $a^{k}\left(\pi^{\star} E\right)$ of $\pi^{\star} E$ by $s_{\Delta}$. More precisely, let $W_{0}=E \backslash X$ and let $W_{1}$ be a neighbourhood of $X$ in $E$, and consider the covering $\mathcal{W}=\left\{W_{0}, W_{1}\right\}$ of $E$. For a $(1,0)$-connection $\nabla$ for $\pi^{\star} E$, we denote by $a^{k}(\nabla)$ the $k$-th Atiyah form of $\nabla$, namely, $a^{k}(\nabla)=\left(\frac{\sqrt{-1}}{2 \pi}\right)^{k} \sigma_{k}\left(K^{1,1}\right)$, where $K^{1,1}$ is the $(1,1)$-component of the curvature seen as a 2 -form with values in $\operatorname{Hom}(E, E)$ and $\sigma_{k}$ denotes the $k$-th elementary symmetric polynomial, see [43, Section 5] for more details. The class $a^{k}\left(\pi^{\star} E\right)$ is represented in $H_{\bar{\partial}}^{k, k}(E) \simeq H_{\bar{D}}^{k, k}(\mathcal{W})$ by the triple $a^{k}\left(\nabla_{\star}\right)=\left(a^{k}\left(\nabla_{0}\right), a^{k}\left(\nabla_{1}\right), a^{k}\left(\nabla_{0}, \nabla_{1}\right)\right)$, where $\nabla_{i}$ is a $(1,0)$-connection for $\pi^{\star} E$ on $W_{i}, i=0,1$, and $a^{k}\left(\nabla_{0}, \nabla_{1}\right)$ is the difference form of $\nabla_{0}$ and $\nabla_{1}$. If we take $\nabla_{0}$ to be $s_{\Delta^{-}}$trivial, we have the vanishing $a^{k}\left(\nabla_{0}\right)=0$ and $a^{k}\left(\nabla_{\star}\right)$ defines a class in $H_{\bar{D}}^{k, k}\left(\mathcal{W}, W_{0}\right)=H_{\bar{D}}^{k, k}(E, E \backslash X)$ that is the Dolbeault-Thom class $\bar{\Psi}_{E}$ of $E$.

The Dolbeault-Thom morphism

$$
\bar{T}_{E}: H_{\bar{\partial}}^{p-k, q-k}(X) \longrightarrow H_{\bar{D}}^{p, q}(E, E \backslash X) .
$$

is given by the cup product with $\bar{\Psi}_{E}$, i.e. if $\bar{\Psi}_{E}$ is represented by $\left(\psi_{1}, \psi_{01}\right)$, it is induced by

$$
\theta \mapsto\left(\psi_{1} \wedge \pi^{\star} \theta, \psi_{01} \wedge \pi^{*} \theta\right) .
$$

The inverse of $\bar{T}_{E}$ is given by the $\bar{\partial}$-integration along the fibres of $\pi$ :

$$
\bar{\pi}_{\star}: H_{\bar{D}}^{p, q}(E, E \backslash X) \longrightarrow H_{\bar{\partial}}^{p-k, q-k}(X) .
$$

It is defined as follows. Let $T_{1}$ denote a bundle of discs of complex dimension $k$ in $W_{1}$ and set $T_{01}=-\partial T_{1}$, which is a bundle of spheres of real dimension $2 k-1$ endowed with the orientation opposite to that of the boundary $\partial T_{1}$ of $T_{1}$. Set $\pi_{1}=\left.\pi\right|_{T_{1}}$ and $\pi_{01}=\left.\pi\right|_{T_{01}}$. Then we have the usual integration along the fibres

$$
\left(\pi_{1}\right)_{\star}: A^{r}\left(W_{1}\right) \longrightarrow A^{r-2 k}(X) \text { and }\left(\pi_{01}\right)_{\star}: A^{r-1}\left(W_{01}\right) \longrightarrow A^{r-2 k}(X) .
$$

The map $\left(\pi_{1}\right)_{\star}$ sends a $(p, q)$-form to a $(p-k, q-k)$-form, while, if $\xi_{01}$ is a $(p, q-1)$-form on $W_{01},\left(\pi_{01}\right)_{\star}\left(\xi_{01}\right)$ consists of $(p-k, q-k)$ and $(p-k+1, q-k-1)$-components. We define

$$
\left(\bar{\pi}_{01}\right)_{\star}: A^{p, q-1}\left(W_{01}\right) \longrightarrow A^{p-k, q-k}(X)
$$

by taking the $(p-k, q-k)$-component of $\left(\pi_{01}\right) *\left(\xi_{01}\right)$, then

$$
\bar{\pi}_{\star} \xi=\left(\pi_{1}\right)_{\star} \xi_{1}+\left(\bar{\pi}_{01}\right)_{\star} \xi_{01} .
$$

In this situation,

$$
\bar{\pi}_{\star} \circ \bar{T}_{E}=1 .
$$

Thus $\bar{\pi}_{\star}$ is surjective and $\bar{T}_{E}$ gives a splitting of

$$
0 \longrightarrow \operatorname{ker} \bar{\pi}_{\star} \longrightarrow H_{\bar{D}}^{p, q}(E, E \backslash X) \stackrel{\bar{\pi}_{*}}{\longrightarrow} H_{\bar{\partial}}^{p-k, q-k}(X) \longrightarrow 0 .
$$

For the $\bar{\partial}$-Thom class $\bar{\Psi}_{E} \in H_{\bar{D}}^{k, k}(E, E \backslash X)$, we have $\bar{\pi}_{\star} \bar{\Psi}_{E}=[1] \in H_{\bar{\partial}}^{0,0}(X)$.

\section{$1.5 \partial \bar{\partial}$-Lemma and Hodge structures}

Although these may be well-known to experts, we recall what the $\partial \bar{\partial}$-Lemma means and some alternative ways of saying that for later use.

Let $X$ be a complex manifold. The de Rham complex $\left(A^{\bullet}(X), d\right)$ of $X$ is the single complex associated with the double complex $\left(A^{\bullet, \bullet}(X), \partial, \bar{\partial}\right), d=\partial+\bar{\partial}$. Recall that [16] $X$ satisfies the $\partial \bar{\partial}$-Lemma if

$$
\operatorname{ker} \partial \cap \operatorname{ker} \bar{\partial} \cap \operatorname{im} d=\operatorname{im} \partial \bar{\partial} \text {. }
$$


We describe the above property in terms of filtrations. Note that $A^{\bullet}(X)$ has two natural filtrations. The first filtration on $A^{h}(X)$ is given by

$$
{ }^{\prime} F^{p} A^{h}(X)=\bigoplus_{i=p}^{h} A^{i, h-i}(X) .
$$

It induces a filtration on $H_{d R}^{h}(X)$ by

$$
{ }^{\prime} F^{p} H_{d R}^{h}(X)=\operatorname{ker} d^{h} \cap{ }^{\prime} F^{p} A^{h}(X) / \operatorname{im} d^{h-1} \cap{ }^{\prime} F^{p} A^{h}(X) .
$$

The second filtration on $A^{h}(X)$ is given by

$$
{ }^{\prime \prime} F^{q} A^{h}(X)=\bigoplus_{j=q}^{h} A^{h-j, j}(X)
$$

and it induces a filtration $\left({ }^{\prime \prime} F^{q} H_{d R}^{h}(X)\right)$ on $H_{d R}^{h}(X)$.

Since $\overline{A^{q, p}(X)}=A^{p, q}(X)$, we may identify the filtration $\left(\overline{{ }^{q} F^{q} A^{h}(X)}\right)$ conjugate to $\left({ }^{\prime} F^{q} A^{h}(X)\right)$ with the second filtration: $\overline{F^{q} A^{h}(X)}={ }^{\prime \prime} F^{q} A^{h}(X)$, which leads to the identification

$$
\overline{{ }^{\prime} F^{q} H_{d R}^{h}(X)}={ }^{\prime \prime} F^{q} H_{d R}^{h}(X) .
$$

We say that the filtration $\left({ }^{\prime} F^{p} H_{d R}^{h}(X)\right)$ is a Hodge filtration of weight $h$ if

$$
H_{d R}^{h}(X)=\bigoplus_{p+q=h}{ }^{\prime} F^{p} H_{d R}^{h}(X) \cap \overline{F^{q} H_{d R}^{h}(X)} .
$$

Lemma 2. The filtration $\left({ }^{\prime} F^{p} H_{d R}^{h}(X)\right)$ is a Hodge filtration of weight $h$ if and only if

$$
H_{d R}^{h}(X)={ }^{\prime} F^{p} H_{d R}^{h}(X) \oplus \overline{F^{q} H_{d R}^{h}(X)} \quad \text { for every }(p, q) \text { with } p+q=h+1 .
$$

Moreover, if this is the case, there is a canonical isomorphism

$$
{ }^{\prime} F^{p} H_{d R}^{h}(X) \cap \overline{{ }^{q} F^{q} H_{d R}^{h}(X)} \simeq{ }^{\prime} G^{p} H_{d R}^{h}(X) \quad \text { for every }(p, q) \text { with } p+q=h,
$$

where ${ }^{\prime} G^{p} H_{d R}^{h}(X)={ }^{\prime} F^{p} H_{d R}^{h}(X) / F^{p+1} H_{d R}^{h}(X)$.

Proof. It is rather straightforward to show the equivalence of two expressions for Hodge filtrations. We only indicate a proof of the last statement for later use. In the sequel we denote $H_{d R}^{h}(X)$ by $H^{h}$.

For $c \in{ }^{\prime} F^{p} H^{h}$ we denote by $[c]^{p}$ its class in ${ }^{\prime} G^{p} H^{h}$. We define a morphism

$$
{ }^{\prime} F^{p} H^{h} \cap \overline{{ }^{q} H^{h}} \longrightarrow{ }^{\prime} G^{p} H^{h} \quad \text { by } c \mapsto[c]^{p}
$$

and show that it is an isomorphism. For the surjectivity, take $[c]^{p} \in{ }^{\prime} G^{p} H^{h}, c \in{ }^{\prime} F^{p} H^{h}$. Then we may write uniquely $c=\sum_{i=0}^{h} c^{i, h-i}$ with $c^{i, h-i} \in{ }^{\prime} F^{i} H^{h} \cap \overline{{ }^{\prime} F^{h-i} H^{h}}$. We have $[c]^{p}=\left[c^{\prime}\right]^{p}, c^{\prime}=\sum_{i=0}^{p} c^{i, h-i}$. Since $\sum_{i=p+1}^{h} c^{i, h-i} \in{ }^{\prime} F^{p+1} H^{h} \subset{ }^{\prime} F^{p} H^{h}$, we have $c^{\prime} \in{ }^{\prime} F^{p} H^{h}$. On the other hand, $c^{\prime}$ is also in $\overline{F^{q} H^{h}}$ and $c^{\prime} \mapsto[c]^{p}$. For the injectivity, take $c \in{ }^{\prime} F^{p} H^{h} \cap \overline{{ }^{\prime} F^{q} H^{h}}$ such that $[c]^{p}=0$. This means that $c \in f^{\prime p+1} H^{h} \cap \overline{F^{q} H^{h}}=0$.

The spectral sequence associated with the first filtration of $A^{\bullet}(X)$ is the Frölicher spectral sequence [20], for which we have

$$
E_{1}^{p, q} \simeq H_{\bar{\partial}}^{p, q}(X), \quad E_{\infty}^{p, q} \simeq{ }^{\prime} G^{p} H_{d R}^{p+q}(X),
$$

Proposition 3 ([16]). A complex manifold X satisfies the $\partial \bar{\partial}$-Lemma if and only if the following two conditions hold:

(1) the Frölicher spectral sequence degenerates at $E_{1}$,

(2) the filtration $\left({ }^{\prime} F^{p} H_{d R}^{h}(X)\right)$ is a Hodge filtration of weight $h$ for every $h \geq 0$. 
Note that every element of ${ }^{\prime} G^{p} H_{d R}^{p+q}(X)$ is expressed as $[[\omega]]^{p}$, where $\omega$ is a $d$-closed form in ${ }^{\prime} F^{p} A^{p+q}(X),[\omega]$ is the class of $\omega$ in ${ }^{\prime} F^{p} H_{d R}^{p+q}(X)$ and $[[\omega]]^{p}$ is the class of $[\omega]$ in ${ }^{\prime} G^{p} H_{d R}^{p+q}(X)$. The condition $d \omega=0$ implies that $\bar{\partial} \omega^{p, q}=0$ when we write $\omega=\sum_{i=p}^{p+q} \omega^{i, p+q-i}$.

The condition (1) above is equivalent to saying that, for every $(p, q)$, the assignment $[[\omega]]^{p} \mapsto\left[\omega^{p, q}\right]$ is well-defined and induces an isomorphism

$$
{ }^{\prime} G^{p} H_{d R}^{p+q}(X) \stackrel{\sim}{\longrightarrow} H_{\bar{\partial}}^{p, q}(X) .
$$

Recall that $A^{p, q}(X)=\overline{A^{q, p}(X)}$ and $A^{h}(X)=\bigoplus_{p+q=h} A^{p, q}(X)$. We ask when these relations carry on to the cohomologies.

Definition 4. 1. We say that $X$ admits a Hodge structure of weight $h$, if there exist isomorphisms

$$
H_{\frac{\partial}{\partial}}^{p, q}(X) \simeq \overline{H_{\frac{\partial}{\partial}}^{q, p}(X)}, p+q=h, \quad \text { and } \quad H_{d R}^{h}(X) \simeq \bigoplus_{p+q=h} H_{\frac{\partial}{\partial}}^{p, q}(X) .
$$

2. A Hodge structure as above is said to be natural, if the following conditions hold:

(H1) Every class in $H_{\bar{\partial}}^{p, q}(X), p+q=h$, admits a representative $\omega$ with $\partial \omega=0$ and $\bar{\partial} \omega=0$, i.e., $d \omega=0$. Moreover, the assignment $\omega \mapsto \bar{\omega}$ induces the first isomorphism above.

(H2) Every class in $H_{d R}^{h}(X)$ admits a representative $\omega$ which may be written $\omega=\sum_{p+q=h} \omega^{p, q}$, where $\omega^{p, q}$ is a $(p, q)$-form with $d \omega^{p, q}=0$. Moreover, the assignment $\omega \mapsto\left(\omega^{p, q}\right)_{p+q=h}$ induces the second isomorphism above.

Remark 5. In (H1) above, $\overline{H_{\frac{q}{\partial}}^{q, p}(X)}$ denotes the vector space conjugate to $H_{\bar{\partial}}^{q, p}(X)$, i.e., the vector space with underlying set $H_{\bar{\partial}}^{q, p}(X)$ and the complex multiplication given by $c \cdot \omega=\bar{c} \omega$. We may rephrase (H1) as:

(H1) ${ }^{\prime}$ Every class in $H_{\bar{\partial}}^{p, q}(X), p+q=h$, admits a representative $\omega$ with $\partial \omega=0$ and $\bar{\partial} \omega=0$, i.e., $d \omega=0$. Moreover, the assignment $\omega \mapsto \omega$ induces an isomorphism $H_{\bar{\partial}}^{p, q}(X) \simeq H_{\partial}^{p, q}(X)$.

Remark 6. See [14, Proposition 4.3] for an example of a compact complex manifold with a non-natural Hodge structure.

Proposition 7. A complex manifold $X$ admits a natural Hodge structure of weight $h$ if and only if the following conditions hold:

(i) the morphism $\operatorname{ker} d \cap^{\prime} F^{p} A^{h}(X) \rightarrow A^{p, h-p}(X), \omega \mapsto \omega^{p, h-p}$, induces an isomorphism ${ }^{\prime} G^{p} H_{d R}^{h}(X) \simeq H_{\frac{\partial}{\partial}}^{p, h-p}(X)$ for every $p$,

(ii) $\left.{ }^{\prime} F^{p} H_{d R}^{h}(X)\right)$ is a Hodge filtration on $H_{d R}^{h}(X)$ of weight $h$.

Proof. Suppose $X$ admits the natural Hodge structure of weight $h$. We claim that there is an isomorphism

$$
{ }^{\prime} F^{p} H_{d R}^{h}(X) \simeq \bigoplus_{i=p}^{h} H_{\bar{\partial}}^{i, h-i}(X)
$$

compatible with the one in (H2) in the sense that the following is commutative:

$$
\begin{aligned}
{ }^{\prime} F^{p} H_{d R}^{h}(X) & \simeq \bigoplus_{i=p}^{h} H_{\frac{\partial}{\partial}}^{i, h-i}(X) \\
\cap & \cap \\
H_{d R}^{h}(X) & \stackrel{\sim}{\simeq} \bigoplus_{i=0}^{h} H_{\frac{\partial}{\partial}}^{i, h-i}(X) .
\end{aligned}
$$

For this, take $\theta \in \operatorname{ker} d^{h} \cap{ }^{\prime} F^{p} A^{h}(X)$ and write $\theta=\sum_{i=p}^{h} \theta^{i, h-i}$ with $\theta^{i, h-i} \in A^{i, h-i}(X)$. From $d \theta=0$ and (H1), we see that there exist $\omega^{i, h-i}$ and $\alpha^{i, h-i}$ in $A^{i, h-i}(X), p \leq i \leq h$, such that $d \omega^{i, h-i}=0$ and that

$$
\theta^{i, h-i}=\omega^{i, h-i}+\partial \alpha^{i-1, h-i}+\bar{\partial} \alpha^{i, h-i-1}
$$


where we set $\alpha^{p-1, h-p}=0$. Then we have

$$
\theta=\sum_{i=p}^{h} \omega^{i, h-i}+d \sum_{i=p}^{h} \alpha^{i, h-i-1} .
$$

By (H2), the assignment $\theta \mapsto\left(\omega^{i, h-i}\right)_{i=p}^{h}$ induces a well-defined morphism ${ }^{\prime} F^{p} H_{d R}^{h}(X) \rightarrow \bigoplus_{i=p}^{h} H_{\bar{\partial}}^{i, h-i}(X) \operatorname{com}^{-}$ patible with the isomorphism of (H2). It is obviously injective. The surjectivity follows from (H1) and it is the desired isomorphism.

From (4), we have ' $G^{p} H_{d R}^{h}(X) \simeq H_{\bar{\partial}}^{p, h-p}(X)$ and the correspondence is the one as given in (i).

We also have an isomorphism $\overline{F^{q} H_{d R}^{h}(X)} \simeq \bigoplus_{j=q}^{h} H_{\bar{\partial}}^{h-j, j}(X)$ compatible with the one in (H2). Thus for $(p, q)$ with $p+q=h+1, H_{d R}^{h}(X)={ }^{\prime} F^{p} H_{d R}^{h}(X) \oplus \overline{F^{q} H_{d R}^{h}(X)}$ and we have (ii).

Now we prove the converse. The condition (ii) implies (cf. Lemma 2)

$$
\begin{gathered}
H_{d R}^{h}(X)=\bigoplus_{p+q=r}{ }^{\prime} F^{p} H_{d R}^{h}(X) \cap \overline{{ }^{\prime} F^{q} H_{d R}^{h}(X)} \quad \text { and } \\
{ }^{\prime} F^{p} H_{d R}^{h}(X) \cap \overline{{ }^{\prime} F^{q} H_{d R}^{h}(X)} \simeq{ }^{\prime} G^{p} H_{d R}^{h}(X), \quad h=p+q .
\end{gathered}
$$

From the condition (i) and (6), we have

$$
\begin{aligned}
H_{\bar{\partial}}^{p, q}(X) & \simeq{ }^{\prime} G^{p} H_{d R}^{h}(X) \simeq{ }^{\prime} F^{p} H_{d R}^{h}(X) \cap \overline{{ }^{\prime} F^{q} H_{d R}^{h}(X)} \\
& \simeq \overline{{ }^{\prime} G^{q} H_{d R}^{h}(X)} \simeq \overline{H_{\frac{q}{\partial}}^{q, p}(X)}, \quad h=p+q .
\end{aligned}
$$

We look at the correspondence above. Take $c \in{ }^{\prime} F^{p} H_{d R}^{h}(X) \cap \overline{{ }^{q} F^{q} H_{d R}^{h}(X)}$, we may write $c=\left[\omega_{1}\right]=\left[\omega_{2}\right]$, where $\omega_{1}=\sum_{i=p}^{h} \omega_{1}^{i, h-i} \in{ }^{\prime} F^{p} A^{h}(X), d \omega_{1}=0$ (thus $\bar{\partial} \omega_{1}^{p, q}=0$ ) and $\omega_{2}=\sum_{j=q}^{h} \omega_{1}^{h-j, j} \in \overline{F^{q} A^{h}(X)}, d \omega_{2}=0$ (thus $\left.\partial \omega_{2}^{p, q}=0\right)$. Then the correspondence is given by $\left[\omega_{1}^{p, q}\right] \leftrightarrow\left[\overline{\omega_{2}^{p, q}}\right]$. From $\left[\omega_{1}\right]=\left[\omega_{2}\right]$, we see that there exist $\theta^{p, q-1} \in A^{p, q-1}(X)$ and $\theta^{p-1, q} \in A^{p-1, q}(X)$ such that

$$
\omega_{1}^{p, q}-\omega_{2}^{p, q}=\partial \theta^{p-1, q}+\bar{\partial} \theta^{p, q-1} .
$$

Then $\omega=\omega_{1}^{p, q}-\bar{\partial} \theta^{p, q-1}=\omega_{2}^{p, q}+\partial \theta^{p-1, q}$ is a representative as in (H1). From (5), (6) and the condition (i), we have $(\mathrm{H} 2)$.

Corollary 8. A complex manifold satisfies the $\partial \bar{\partial}$-Lemma if and only if it admits a natural Hodge structure of weight $h$ for every $h$.

Remark 9. If we use the Bott-Chern cohomology $H_{B C}^{\bullet, \bullet}(X)=\frac{\operatorname{ker} \partial \cap \operatorname{ker} \bar{\partial}}{i m \partial \bar{\partial}}$, the condition (3) means that the morphism

$$
H_{B C}^{\bullet, \bullet}(X) \longrightarrow H_{d R}^{\bullet}(X)
$$

induced by the identity is injective. A numerical characterization of the $\partial \bar{\partial}$-Lemma in terms of the dimension of the Bott-Chern cohomology and the Betti numbers is provided in [7] and in [6] using only Bott-Chern numbers.

\section{Dolbeault cohomology of the projectivization of a holomorphic vector bundle}

Let $X$ be a smooth manifold. Also let $\pi: V \rightarrow X$ be a complex vector bundle of rank $k$ and denote by $\rho$ : $\mathbb{P}(V) \rightarrow X$ its projectivization. We may regard $H_{d R}^{\bullet}(\mathbb{P}(V))$ as an $H_{d R}^{\bullet}(X)$-module (in fact, $H_{d R}^{\bullet}(X)$-algebra). Here we regard it as a right module by our convention and the module structure is given by $c \cdot a=c \smile \rho^{\star}(a)$ for 
$c \in H_{d R}^{\bullet}(\mathbb{P}(V))$ and $a \in H_{d R}^{\bullet}(X)$, where $\smile$ denotes the cup product. In the sequel it will be simply denoted by $\cdot$, if there is no fear of confusion.

In the above situation we have the tautological bundle $T$ on $\mathbb{P}(V)$, which is a rank one subbundle of $\rho^{\star} V$ with the universal bundle $Q$ as the quotient so that we have an exact sequence of vector bundles on $\mathbb{P}(V)$ :

$$
0 \longrightarrow T \longrightarrow \rho^{*} V \longrightarrow Q \longrightarrow 0 .
$$

Recall that $\rho^{*} V=\{(v, l) \in V \times \mathbb{P}(V) \mid \pi(v)=\rho(l)\}$. We may think of a point $l$ in $\mathbb{P}(V)$ as a line in $V_{x}=\mathbb{C}^{k}$, $x=\pi(v)$, and we have $T=\left\{(v, l) \in \rho^{\star} V \mid v \in l\right\}$.

We recall the following, which is a direct consequence of the Leray-Hirsch theorem (cf. [22, Proposition page 606], [47, Lemma 7.32]):

Proposition 10. In the above situation, $H_{d R}^{\bullet}(\mathbb{P}(V))$ is a free $H_{d R}^{\bullet}(X)$-module with basis $1, \gamma, \ldots, \gamma^{k-1}$, where $\gamma=c^{1}(T)$ is the first Chern class of $T$.

The essential point in the above is that the restriction of $\gamma$ to each fibre, which is the projective space $\mathbb{P}^{k-1}$, is the first Chern of the tautological bundle (dual of the hyperplane bundle) on $\mathbb{P}^{k-1}$ and that their powers up to the $(k-1)$-st form a $\mathbb{C}$-basis of $H_{d R}^{\cdot}\left(\mathbb{P}^{k-1}\right)$. As an $H_{d R}^{\cdot}(X)$-algebra, $H_{d R}^{\cdot}(\mathbb{P}(V))$ is generated by $\gamma$ with the single relation

$$
\sum_{i=0}^{k}(-1)^{i} \gamma^{i} \cdot \rho^{\star} c^{k-i}(V)=0,
$$

where $c^{k-i}(V)$ is the $(k-i)$-th Chern class of $V$. The relation can be seen from $c(T) \cdot c(Q)=\rho^{*} c(V)$, the relation among the total Chern classes, which follows from (7).

If we take a metric connection for $T$, its curvature form $\kappa$ is of type $(1,1)$ and is simultaneously $d$ - and $\bar{\partial}$-closed. We also have $\bar{\kappa}=-\kappa$. The class of $\frac{\sqrt{-1}}{2 \pi} \kappa$ in $H_{d R}^{2}(\mathbb{P}(V))$ is the first Chern class $\gamma=c^{1}(T)$ and its class in $H_{\bar{\partial}}^{1,1}(\mathbb{P}(V))$ is the first Atiyah class $a^{1}(T)$. Note that they cannot be compared directly on the cohomology level, in general. However, their restrictions to each fibre of $\mathbb{P}(V) \rightarrow X$ may be identified, as the fibre is $\mathbb{P}^{k-1}$ and it satisfies the $\partial \bar{\partial}$-Lemma.

Proposition 11. Let $V \rightarrow X$ be a holomorphic vector bundle of rank $k$ on a compact complex manifold $X$. Then $H_{\bar{\partial}}^{\bullet \bullet \bullet}(\mathbb{P}(V))$ is a free $H_{\bar{\partial}}^{\bullet, \bullet}(X)$-module with basis $1, \alpha, \ldots, \alpha^{k-1}$, where $\alpha=a^{1}(T)$ is the first Atiyah class of $T$.

Proof. By [15, Lemma 18], we see that $H_{\bar{\partial}}^{\bullet \bullet \bullet}(\mathbb{P}(V))$ is generated by $\alpha$ as an $H_{\bar{\partial}}^{\bullet \bullet \bullet}(X)$-algebra. We have a relation as (8), replacing $\gamma$ and $c^{k-i}(V)$ with $\alpha$ and $a^{k-i}(V)$, the $(k-i)$-th Atiyah class of $V$, from which we see that $1, \alpha, \ldots, \alpha^{k-1}$ generate $H_{\bar{\partial}}^{\bullet \bullet \bullet}(\mathbb{P}(V))$ as an $H_{\bar{\partial}}^{\bullet, \bullet}(X)$-module. The proposition follows from the following:

Claim. $1, \alpha, \ldots, \alpha^{k-1}$ are linearly independent over $H_{\bar{\partial}}^{\bullet, \bullet}(X)$.

To prove this, we look at the $\mathbb{C}$-algebra structure of $H_{\bar{\partial}}^{\bullet \bullet \bullet}(\mathbb{P}(V))$. Let $n=\operatorname{dim} X$ and $h^{p, q}=\operatorname{dim} H_{\frac{\partial}{\partial}}^{p, q}(X)$. For each $(p, q)$ with $h^{p, q} \neq 0$, we take a basis $\left\{u_{i}^{p, q}\right\}_{1 \leq i \leq h}$ of $H_{\bar{\partial}}^{p, q}(X)$ so that $\left\{u_{i^{\prime}}^{n-p, n-q}\right\}_{1 \leq i^{\prime} \leq h}$ is the basis of $H_{\bar{\partial}}^{n-p, n-q}(X)$ dual to $\left\{u_{i}^{p, q}\right\}$ via the Kodaira-Serre duality, $h=h^{p, q}=h^{n-p, n-q}$ :

$$
\int_{X} u_{i}^{p, q} \cdot u_{i^{\prime}}^{n-p, n-q}= \pm \delta_{i i^{\prime}} .
$$

Obviously $\left\{\alpha^{r} \cdot \rho^{\star} u_{i}^{p, q}\right\}_{0 \leq r \leq k-1, p, q, i}$ span the $\mathbb{C}$-vector space $H_{\frac{\bullet}{\partial}}^{\bullet \bullet}(\mathbb{P}(V))$. We show that they are linearly independent over $\mathbb{C}$, which will prove the claim and the proposition. For this we introduce a relation $>$ in the set $\Lambda$ of indices $\lambda=(r, p, q, i)$ by saying that $\left(r_{1}, p_{1}, q_{1}, i_{1}\right)>\left(r_{2}, p_{2}, q_{2}, i_{2}\right)$ if one of the following holds:

1. $2 r_{1}+p_{1}+q_{1}>2 r_{2}+p_{2}+q_{2}$,

2. $2 r_{1}+p_{1}+q_{1}=2 r_{2}+p_{2}+q_{2}$ and $p_{1}+q_{1}>p_{2}+q_{2}$,

3. $r_{1}=r_{2}, p_{1}+q_{1}=p_{2}+q_{2}$ and $p_{1}>p_{2}$,

4. $r_{1}=r_{2}, p_{1}=p_{2}, q_{1}=q_{2}$ and $i_{1}>i_{2}$. 
With this, $\Lambda$ becomes a totally ordered set. Let $\Lambda^{\prime}$ denote the set $\Lambda$ with the order defined by reversing the inequalities in (1), (2) and (3) and keeping that in (4) above. We consider the matrix $\left(v_{\lambda} \cdot v_{\lambda^{\prime}}\right)_{\left(\lambda, \lambda^{\prime}\right) \in \Lambda \times \Lambda^{\prime}}$, where, for $\lambda=(r, p, q, i), v_{\lambda}=\alpha^{r} \cdot \rho^{*} u_{i}^{p, q}$ and similarly for $v_{\lambda^{\prime}}$. On the diagonal, we have $v_{\lambda} \cdot v_{\lambda^{\prime}}$ for which $r+r^{\prime}=k-1$, $p+p^{\prime}=n, q+q^{\prime}=n$ and $i=i^{\prime}$ (note that this makes sense as $p+p^{\prime}=n$ and $q+q^{\prime}=n$ ), when we write $\lambda=(r, p, q, i)$ and $\lambda^{\prime}=\left(r^{\prime}, p^{\prime}, q^{\prime}, i^{\prime}\right)$. In this case, noting that $\rho_{\star} \alpha^{k-1}=(-1)^{k-1}$, as the restriction of $\alpha$ to each fibre is the first Atiyah class of the dual of the hyperplane bundle, by the projection formula,

$$
\int_{\mathbb{P}(V)}\left(\alpha^{r} \cdot \rho^{\star} u_{i}^{p, q}\right) \cdot\left(\alpha^{r^{\prime}} \cdot \rho^{\star} u_{i^{\prime}}^{p^{\prime}, q^{\prime}}\right)=\rho \star \alpha^{k-1} \cdot \int_{X} u_{i}^{p, q} \cdot u_{i}^{n-p, n-q}= \pm 1 .
$$

On the upper triangle, but off the diagonal, we have $v_{\lambda} \cdot v_{\lambda^{\prime}}$ for which one of the following holds:

1. $2 r+2 r^{\prime}+p+p^{\prime}+q+q^{\prime}>2(n+k-1)$,

2. $2 r+2 r^{\prime}+p+p^{\prime}+q+q^{\prime}=2(n+k-1)$ and $p+p^{\prime}+q+q^{\prime}>2 n$,

3. $r+r^{\prime}=k-1, p+p^{\prime}+q+q^{\prime}=2 n$ and $p+p^{\prime}>n$,

4. $r+r^{\prime}=k-1, p+p^{\prime}=n, q+q^{\prime}=n$ and $i<i^{\prime}$.

Recalling that $\operatorname{dim} X=n$ and $\operatorname{dim} \mathbb{P}(V)=n+k-1$, we have, in the case (1), (2) or (3),

$$
\left(\alpha^{r} \cdot \rho^{\star} u_{i}^{p, q}\right) \cdot\left(\alpha^{r^{\prime}} \cdot \rho^{\star} u_{i^{\prime}}^{p^{\prime}}, q^{\prime}\right)=0,
$$

by dimension reason. In the case (4), $\int_{\mathbb{P}(V)} v_{\lambda} \cdot v_{\lambda^{\prime}}=0$ by a similar computation as above. Thus the KodairaSerre dual of the matrix $\left(v_{\lambda} \cdot v_{\lambda^{\prime}}\right)$ is triangular with \pm 1 's along the diagonal, which shows that the $\alpha^{r} \cdot \rho^{\star} u_{i}^{p, q}$, are linearly independent over $\mathbb{C}$.

Corollary 12. Let $X$ be a compact complex manifold and $V \rightarrow X$ a holomorphic fibre bundle on $X$. If $X$ satisfies the $\partial \bar{\partial}$-lemma, so does $\mathbb{P}(V)$.

Proof. The statement follows from Corollary 8 and Propositions 10 and 11, noting that $\gamma$ and $\alpha$ are both represented by the same form $\frac{\sqrt{-1}}{2 \pi} \kappa$ as above.

\section{Hodge structures under blow-ups}

We can now prove explcit expressions for the de Rham (Proposition 16) and Dolbeault (Proposition 19) cohomologies of the blow-up and then Theorem 13. Compare also [50, Theorem 1.3] for similar results using Bott-Chern cohomology, and [40, Corollary 25] for a clear statement and argument.

Let $X$ be a compact complex manifold of dimension $n$ and $Z$ a closed complex submanifold of codimension $k$. Also let $\tau: \tilde{X}:=\tilde{X}_{Z} \rightarrow X$ be the blow-up of $X$ along $Z$ with exceptional divisor $\mathrm{E}=\mathbb{P}\left(N_{Z \mid X}\right)$. Here we assume that

$$
Z \text { admits a holomorphically contractible neighbourhood }
$$

that is, there exists $U \supset Z$ with $r: U \rightarrow Z$ holomorphic and $\left.r\right|_{Z}=$ id. In this case $\mathrm{E}$ also admits a holomorphically contractible neighbourhood $\tilde{U} \supset \mathrm{E}$ with $\tilde{r}: \tilde{U} \rightarrow \mathrm{E}$ holomorphic and $\left.\tilde{r}\right|_{\mathrm{E}}=\mathrm{id}$. Thus we have the following diagram:

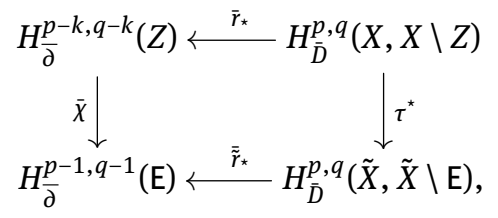

where the horizontal arrows are the $\bar{\partial}$-integrations along the fibres, $\tau^{\star}$ is the morphism induced by $\tau$ and $\bar{\chi}$ is given by $z \mapsto a^{k-1}(Q) \cdot \tau_{\mathrm{E}}^{\star} z$, see the proof below for details. Here we spend some words to clarify the 
heavy notation: accordingly with [43], the bar refers to the holomorphic aspects of the theory, while the tilde concerns to the level of the blow-up.

We do not know whether or not the diagram (10) is commutative. The first condition in (11) below is apparently weaker than the commutativity (cf. Remark 20. (5) below).

Theorem 13. Let $X$ be a compact complex manifold and $Z$ a closed submanifold of $X$. Also let $\tau: \tilde{X}_{Z} \rightarrow X$ be the blow-up of $X$ along $Z$. Assume that the conditions (9) above and

$$
\operatorname{im} \overline{\tilde{r}}_{\star} \circ \tau^{\star} \subset \operatorname{im} \bar{\chi}, \quad \operatorname{ker} \overline{\tilde{r}}_{\star} \subset \operatorname{im} \tau^{*}
$$

hold. Then, if both $X$ and $Z$ admit a Hodge structure, so does $\tilde{X}_{Z}$.

Proof. Algebraic preliminaries. We quote the following lemma, see for instance [11, Lemme II.6]:

Lemma 14. Let $R$ be a commutative ring with unity and let

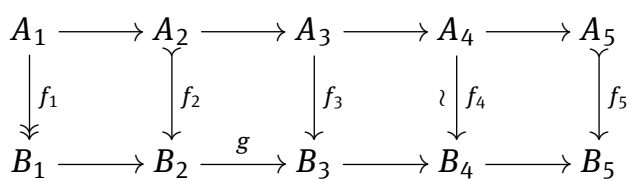

be a commutative diagram of $R$-modules with exact rows such that $f_{1}$ is surjective, $f_{2}$ and $f_{5}$ are injective and $f_{4}$ is an isomorphism. Then $f_{3}$ is injective and $g$ induces an isomorphism

$$
\tilde{g}: B_{2} / f_{2} A_{2} \stackrel{\sim}{\longrightarrow} B_{3} / f_{3} A_{3} .
$$

In the above situation, we have the diagram with an exact row:

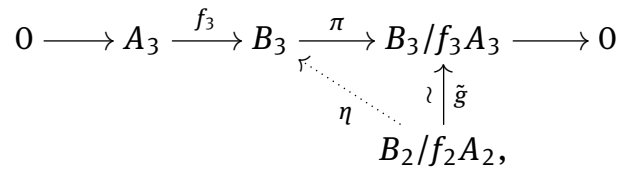

where $\pi$ is the canonical surjection. If there is a splitting $\eta: B_{2} / f_{2} A_{2} \rightarrow B_{3}$, i.e., a morphism with $(\tilde{g})^{-1} \circ \pi \circ \eta=$ id, we have an isomorphism

$$
A_{3} \oplus\left(B_{2} / f_{2} A_{2}\right) \stackrel{\sim}{\longrightarrow} B_{3}, \quad(a,[b]) \mapsto f_{3}(a)+\eta([b]) .
$$

Note that the isomorphism depends on the splitting.

In the sequel, we try to express the cohomology of $\tilde{X}$ in terms of those of $X$ and $Z$ using the above.

de Rham cohomology. Let us start with the de Rham case. Note that, for this case, the assumption (9) (or (11)) is not necessary; for the map $r$, simply take the one given by the Tubular Neighbourhood Theorem, although it is only smooth that is sufficient.

Considering the exact sequence (1) for the pairs $(X, X \backslash Z)$ and $(\tilde{X}, \tilde{X} \backslash \mathrm{E})$, we have the commutative diagram with exact rows:

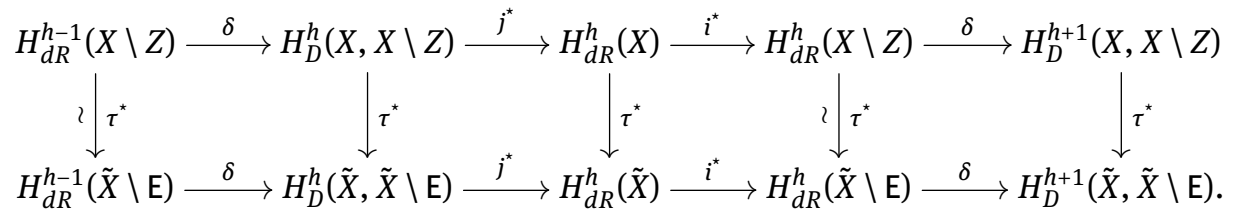

We study the morphism $\tau^{\star}: H_{D}^{\bullet}(X, X \backslash Z) \rightarrow H_{D}^{\bullet}(\tilde{X}, \tilde{X} \backslash \mathrm{E})$ more closely. First, it is injective by [45, Theorem 3.2] and Lemma 14 shows that $\tau^{\star}: H_{d R}^{h}(X) \rightarrow H_{d R}^{h}(\tilde{X})$ is injective (in fact this is already implied by [51, Theorem 3.1]) and that $j^{*}$ in the second row induces an isomorphism

$$
H_{D}^{h}(\tilde{X}, \tilde{X} \backslash \mathrm{E}) / \tau^{*} H_{D}^{h}(X, X \backslash Z) \stackrel{\sim}{\longrightarrow} H_{d R}^{h}(\tilde{X}) / \tau^{\star} H_{d R}^{h}(X) .
$$


We try to express the left hand side in terms of the cohomologies of $Z$ and $\mathrm{E}$ and along the way we reprove the injectivity of $\tau^{\star}$ on the relative cohomology (cf. Remark 20. (1) below).

Let $\pi: N:=N_{Z \mid X} \rightarrow Z$ be the normal bundle of $Z$ in $X$. Recall that $\mathrm{E}$ is the projectivization $\mathbb{P}(N)$ of $N$ and that $\tau_{\mathrm{E}}:=\left.\tau\right|_{\mathrm{E}}: \mathrm{E}=\mathbb{P}(N) \rightarrow Z$ is the projection of the bundle. The normal bundle of $\mathrm{E}$ in $\tilde{X}$ is the tautological bundle $\tilde{\pi}: T \rightarrow \mathrm{E}=\mathbb{P}(N)$. It is a subbundle of $\tau_{\mathrm{E}}^{\star} N$ with the universal bundle $Q$ as the quotient so that we have an exact sequence of vector bundles on $E$ (cf. (7)):

$$
0 \longrightarrow T \stackrel{\iota}{\longrightarrow} \tau_{\mathrm{E}}^{\star} N \longrightarrow Q \longrightarrow 0 .
$$

Recall that $\tau_{\mathrm{E}}^{\star} N=\left\{(v, e) \in N \times \mathrm{E} \mid \pi(v)=\tau_{\mathrm{E}}(e)\right\}$ so that we have the commutative diagram

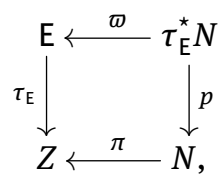

where $p$ and $\varpi$ denote the restrictions of the projections onto the first and the second factors, respectively.

Let $\varphi: U \stackrel{\sim}{\rightarrow} W$ be a diffeomorphism as given by the Tubular Neighbourhood Theorem, with $U$ and $W$ neighbourhoods of $Z$ in $X$ and $N$, respectively. We set $r=\pi \circ \varphi: U \rightarrow Z$. We may choose neighbourhoods $\tilde{U}$ and $\tilde{W}$ of $\mathrm{E}$ in $\tilde{X}$ and $T$ and a diffeomorphism $\tilde{\varphi}: \tilde{U} \stackrel{\sim}{\rightarrow} \tilde{W}$ so that $\tilde{U}=\tau^{-1} U$ and $\varphi \circ \tau \circ(\tilde{\varphi})^{-1}: \tilde{W} \rightarrow W$ is equal to $\left.p \circ \iota\right|_{\tilde{W}}$. We set $\tilde{r}=\tilde{\pi} \circ \tilde{\varphi}: \tilde{U} \rightarrow$ E so that we have the commutative diagram

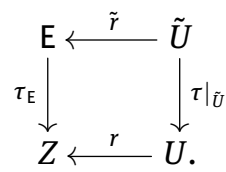

We have the Thom class $\Psi_{Z} \in H_{D}^{2 k}(X, X \backslash Z)=H_{D}^{2 k}(U, U \backslash Z)$ of $Z$ and that $\Psi_{\mathrm{E}} \in H_{D}^{2}(\tilde{X}, \tilde{X} \backslash \mathrm{E})=H_{D}^{2}(\tilde{U}, \tilde{U} \backslash \mathrm{E})$ of $E$.

Lemma 15. In the above situation, we have

$$
\tau^{\star} \Psi_{Z}=\Psi_{\mathrm{E}} \smile \tilde{r}^{\star} c^{k-1}(Q),
$$

where $c^{k-1}(Q)$ is the top Chern class of $Q$.

Proof of Lemma 15. Noting that $r \circ \tau=\tau_{\mathrm{E}} \circ \tilde{r}$, we have the exact sequence of vector bundles on $\tilde{U}$ :

$$
0 \longrightarrow \tilde{r}^{\star} T \longrightarrow \tau^{\star} r^{\star} N \longrightarrow \tilde{r}^{\star} Q \longrightarrow 0 .
$$

Let $s_{\Delta}$ and $\tilde{s}_{\Delta}$ denote the diagonal sections of $\pi^{\star} N$ on $N$ and of $\tilde{\pi}^{\star} T$ on $T$, respectively. We denote the corresponding sections of $r^{\star} N$ on $U$ and of $\tilde{r}^{\star} T$ on $\tilde{U}$ by $s$ and $\tilde{s}$. We claim that $\tilde{s}$ is mapped to $\tau^{\star} s$ by the first morphism above. To see this, first note that $s_{\Delta}(v)=(v, v)$, where we think of the first component as the fibre component. The section $s$ is given by, for $x \in U, s(x)=\varphi(x) \in\left(r^{\star} N\right)_{x}=N_{z}, z=r(x)=\pi \circ \varphi(x)$. On the other hand $\tilde{s}_{\Delta}(t)=(t, t)$ and $\tilde{s}$ is given by, for $\tilde{x} \in \tilde{U}, \tilde{s}(\tilde{x})=\tilde{\varphi}(\tilde{x}) \in\left(\tilde{r}^{\star} T\right)_{\tilde{x}}=T_{e}, e=\tilde{r}(\tilde{x})=\tilde{\pi} \circ \tilde{\varphi}(\tilde{x})$. We have $\tau^{\star} s(\tilde{x})=s(\tau(\tilde{x}))=\varphi \circ \tau(\tilde{x})=p \circ \iota \circ \tilde{\varphi}(\tilde{x})$, which proves the claim.

Recall that $\Psi_{Z}$ is the localization of $c^{k}\left(r^{\star} N\right)$ by $s$ so that $\tau^{\star} \Psi_{Z}$ is the localization of $c^{k}\left(\tau^{\star} r^{\star} N\right)$ by $\tau^{\star} s$. The latter can be described as follows. Let $\tilde{\nabla}_{0}$ be an $\tilde{s}$-trivial connection for $\tilde{r}^{\star} T$ on $\tilde{U}_{0}$ and let $\nabla^{Q}$ be a connection for $Q$ on E. Then there exists a $\tau^{\star} s$-trivial connection $\nabla_{0}$ for $\tau^{\star} r^{\star} N$ on $\tilde{U}_{0}$ such that $\left(\tilde{\nabla}_{0}, \nabla_{0}, \tilde{r}^{\star} \nabla^{Q}\right)$ is compatible with (14) on $\tilde{U}_{0}$. Let $\tilde{\nabla}_{1}$ be an arbitrary connection for $\tilde{r}^{\star} T$ on $\tilde{U}$. Then there exists a connection $\nabla_{1}$ for $\tau^{\star} r^{\star} N$ on $\tilde{U}$ such that $\left(\tilde{\nabla}_{1}, \nabla_{1}, \tilde{r}^{\star} \nabla^{Q}\right)$ is compatible with (14) on $\tilde{U}$. Then $\tau^{\star} \Psi_{Z}$ is represented by

$$
\left(c^{k}\left(\nabla_{1}\right), c^{k}\left(\nabla_{0}, \nabla_{1}\right)\right)=\left(c^{1}\left(\tilde{\nabla}_{1}\right) \cdot \tilde{r}^{*} c^{k-1}\left(\nabla^{Q}\right), c^{1}\left(\tilde{\nabla}_{0}, \tilde{\nabla}_{1}\right) \cdot \tilde{r}^{\star} c^{k-1}\left(\nabla^{Q}\right)\right) .
$$

Since $\left(c^{1}\left(\tilde{\nabla}_{1}\right), c^{1}\left(\tilde{\nabla}_{0}, \tilde{\nabla}_{1}\right)\right)$ represents $\Psi_{\mathrm{E}}$, we have the lemma. 
From the above lemma, we see that the following diagram is commutative:

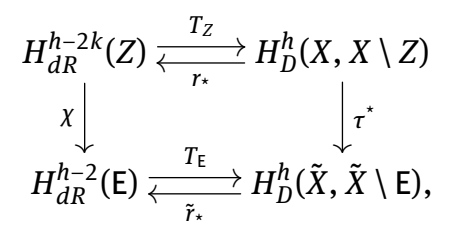

where $\chi$ is the morphism given by $z \mapsto c^{k-1}(Q) \smile \tau_{\mathrm{E}}^{\star} z$. In the above $T_{Z}$ and $r$ are isomorphisms and the inverses of each other, similarly for $T_{\mathrm{E}}$ and $\tilde{r}_{\star}$. Thus $\chi$ is injective and $T_{\mathrm{E}}$ induces an isomorphism

$$
H_{d R}^{h-2}(\mathrm{E}) / \chi H_{d R}^{h-2 k}(Z) \stackrel{\sim}{\longrightarrow} H_{D}^{h}(\tilde{X}, \tilde{X} \backslash \mathrm{E}) / \tau^{\star} H_{D}^{h}(X, X \backslash Z) .
$$

Now we study the left hand side. We claim that $H_{d R}^{\bullet}(\mathrm{E})$ is a free $H_{d R}^{\bullet}(Z)$-module with basis $1, \gamma, \ldots, \gamma^{k-2}, c^{k-1}(Q), \gamma=c^{1}(T)$. To see this, from (13) we have the relation $c(T) \cdot c(Q)=\tau_{\mathrm{E}}^{\star} c(N)$ among the total Chern classes. Thus $c(Q)=c(T)^{-1} \cdot \tau_{\mathrm{E}}^{\star} c(N)$ and we have

$$
c^{k-1}(Q)=\sum_{i=0}^{k-2}(-1)^{i} \gamma^{i} \cdot \tau_{\mathrm{E}}^{\star} c^{k-1-i}(N)+(-1)^{k-1} \gamma^{k-1},
$$

which proves the claim in view of Proposition 10. Thus we have

$$
H_{d R}^{h-2}(\mathrm{E}) / \chi H_{d R}^{h-2 k}(Z) \simeq \bigoplus_{i=0}^{k-2} \gamma^{i} \cdot \tau_{\mathrm{E}}^{\star} H_{d R}^{h-2 i-2}(Z) \subset H_{d R}^{h-2}(\mathrm{E}) .
$$

By (12), (16) and (18), we have the diagram:

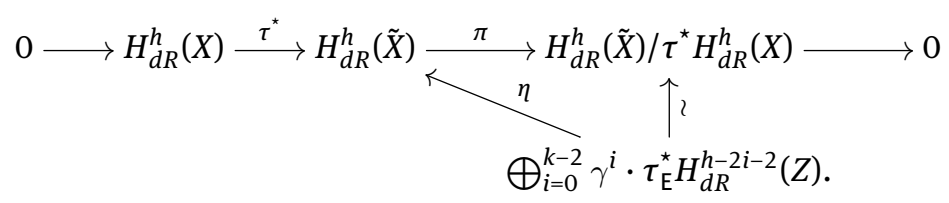

The restriction of the Gysin morphism $\left(i_{\mathrm{E}}\right)_{\star}=j^{\star} \circ T_{\mathrm{E}}: H_{d R}^{h-2}(\mathrm{E}) \rightarrow H_{d R}^{h}(\tilde{X})$ gives a splitting $\eta$ and we have:

Proposition 16. There is an isomorphism

$$
H_{d R}^{h}(X) \oplus \bigoplus_{i=0}^{k-2} H_{d R}^{h-2 i-2}(Z) \stackrel{\sim}{\longrightarrow} H_{d R}^{h}(\tilde{X}),
$$

which is given by $\left(x,\left(z_{i}\right)_{i=0}^{k-2}\right) \mapsto \tau^{\star} x+\sum_{i=0}^{k-2}\left(i_{\mathrm{E}}\right) \star\left(\gamma^{i} \cdot \tau_{\mathrm{E}}^{\star} z_{i}\right)$ for $x \in H_{d R}^{h}(X)$ and $z_{i} \in H_{d R}^{h-2 i-2}(Z)$.

Dolbeault cohomology. Considering the exact sequence (2) for the pairs $(X, X \backslash Z)$ and $(\tilde{X}, \tilde{X} \backslash \mathrm{E})$, we have the commutative diagram with exact rows:

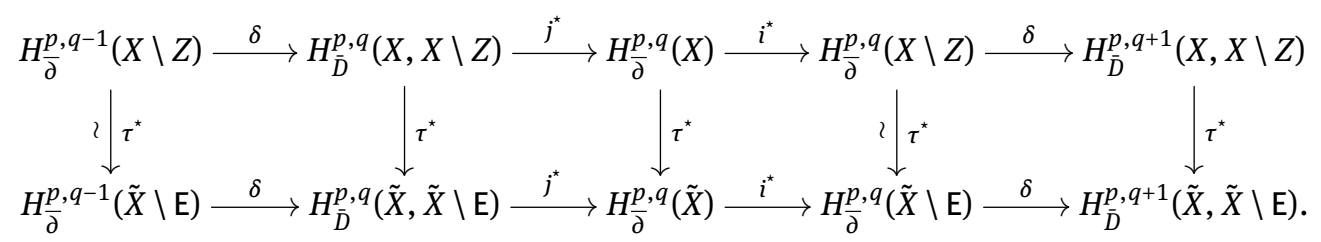

The essential difference from the de Rham case occurs for the relative cohomology and the morphism $\tau^{\star}: H_{\bar{D}}^{p, q}(X, X \backslash Z) \rightarrow H_{\bar{D}}^{p, q}(\tilde{X}, \tilde{X} \backslash E)$, which we are going to analyze. First, it is injective by [45, Theorem 3.1] and Lemma 14 shows that $\tau^{\star}: H_{\bar{\partial}}^{p, q}(X) \rightarrow H_{\bar{\partial}}^{p, q}(\tilde{X})$ is injective (again this is already implied by [51, Theorem 3.1]) and that $j^{\star}$ in the second row induces an isomorphism

$$
H_{\tilde{D}}^{p, q}(\tilde{X}, \tilde{X} \backslash \mathrm{E}) / \tau^{\star} H_{\bar{D}}^{p, q}(X, X \backslash Z) \stackrel{\sim}{\longrightarrow} H_{\bar{\partial}}^{p, q}(\tilde{X}) / \tau^{\star} H_{\bar{\partial}}^{p, q}(X) .
$$


We try to express the left hand side in terms of cohomologies of $Z$ and $\mathrm{E}$.

Recall that the normal bundle $\pi: N \rightarrow Z$ of $Z$ is a holomorphic vector bundle of rank $k$ on $Z$. By the assumption (9), we see that there exist neighbourhoods $U$ and $W$ of $Z$ in $X$ and $N$, respectively, and a biholomorphic map $\varphi: U \rightarrow W$ so that $r=\pi \circ \varphi: U \rightarrow Z$. Thus we have isomorphisms

$$
H_{\bar{D}}^{p, q}(X, X \backslash Z) \simeq H_{\bar{D}}^{p, q}(U, U \backslash Z) \underset{\varphi^{*}}{\sim} H_{\bar{D}}^{p, q}(W, W \backslash Z) \simeq H_{\bar{D}}^{p, q}(N, N \backslash Z),
$$

where the first and the last isomorphisms are excisions. The $\bar{\partial}$-Thom class $\bar{\Psi}_{Z}$ of $Z$ is, by definition, the class in $H_{\bar{D}}^{k, k}(X, X \backslash Z)$ that corresponds to $\bar{\Psi}_{N}$ by the above isomorphism. We have the $\bar{\partial}$-Thom morphism

$$
\bar{T}_{Z}: H_{\bar{\partial}}^{p-k, q-k}(Z) \longrightarrow H_{\bar{D}}^{p, q}(U, U \backslash Z)=H_{\bar{D}}^{p, q}(X, X \backslash Z),
$$

which is given by $z \mapsto \bar{\Psi}_{Z} \smile r^{\star} z$. It gives a splitting of

$$
0 \longrightarrow \operatorname{ker} \bar{r}_{\star} \longrightarrow H_{\bar{D}}^{p, q}(X, X \backslash Z) \stackrel{\bar{r}_{\star}}{\longrightarrow} H_{\bar{\partial}}^{p-k, q-k}(Z) \longrightarrow 0 .
$$

Under the assumption (9), E also admits a holomorphic retraction $\tilde{r}: \tilde{U} \rightarrow \mathrm{E}, \tilde{U}=\tau^{-1} U$, such that the following diagram is commutative:

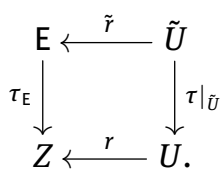

Thus we have the $\bar{\partial}$-Thom class $\bar{\Psi}_{\mathrm{E}} \in H_{\bar{D}}^{1,1}(\tilde{X}, \tilde{X} \backslash \mathrm{E})=H_{\bar{D}}^{1,1}(\tilde{U}, \tilde{U} \backslash \mathrm{E})$ of $\mathrm{E}$ and the $\bar{\partial}$-Thom morphism

$$
\bar{T}_{\mathrm{E}}: H_{\bar{\partial}}^{p-1, q-1}(\mathrm{E}) \longrightarrow H_{\bar{D}}^{p, q}(\tilde{U}, \tilde{U} \backslash \tilde{Z})=H_{\bar{D}}^{p, q}(\tilde{X}, \tilde{X} \backslash \mathrm{E}),
$$

which is given by $a \mapsto \bar{\Psi}_{\mathrm{E}} \smile \tilde{r}^{\star} a$. It gives a splitting of

$$
0 \longrightarrow \operatorname{ker} \overline{\tilde{r}}_{\star} \longrightarrow H_{\bar{D}}^{p, q}(\tilde{X}, \tilde{X} \backslash \mathrm{E}) \stackrel{\overline{\tilde{r}}_{\star}}{\longrightarrow} H_{\bar{\partial}}^{p-1, q-1}(\mathrm{E}) \longrightarrow 0 .
$$

We have the following lemma, which is the holomorphic analogue of Lemma 15 and is proven by the same argument with de Rham cohomology and Chern classes are replaced by Dolbeault cohomology and Atiyah classes, respectively:

Lemma 17. We have:

$$
\tau^{\star} \bar{\Psi}_{Z}=\bar{\Psi}_{\mathrm{E}} \smile \tilde{r}^{\star} a^{k-1}(Q),
$$

where $a^{k-1}(Q)$ denotes the top Atiyah class of $Q$.

From the above lemma, we see that the following diagrams are commutative:
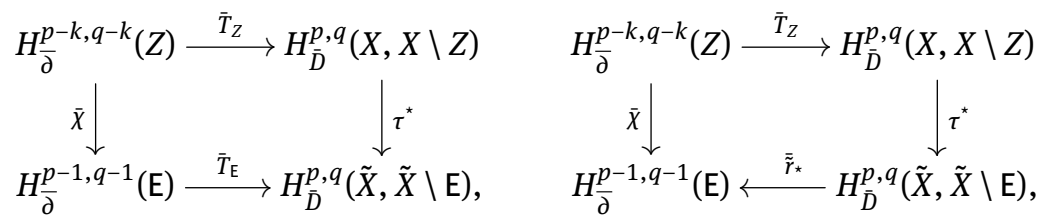

where $\bar{\chi}$ is the morphism given by $z \mapsto a^{k-1}(Q) \cup \tau_{\mathrm{E}}^{\star} z$.

From the first commutative diagram above, $\bar{T}_{\tilde{Z}}$ induces a well-defined morphism

$$
\psi: H_{\bar{\partial}}^{p-1, q-1}(\mathrm{E}) / \bar{\chi} H_{\bar{\partial}}^{p-k, q-k}(Z) \longrightarrow H_{\bar{D}}^{p, q}(\tilde{X}, \tilde{X} \backslash \mathrm{E}) / \tau^{\star} H_{\bar{D}}^{p, q}(X, X \backslash Z) .
$$

Proposition 18. Under the assumption (11), $\psi$ is an isomorphism.

Proof of Proposition 18. For the surjectivity, take $[\tilde{c}], \tilde{c} \in H_{\bar{D}}^{p, q}(\tilde{X}, \tilde{X} \backslash \mathrm{E})$. We may write $\tilde{c}=\bar{T}_{\tilde{Z}}(\tilde{a})+\rho, \rho \in \operatorname{ker} \overline{\tilde{r}}_{\star}$. Thus by the second condition in (11), $\psi([\tilde{a}])=[\tilde{c}]$. For the injectivity, take $[\tilde{a}]$ such that $T_{\tilde{Z}}(\tilde{a})=\tau^{\star}(c)$ for some $c \in H_{\bar{D}}^{p, q}(X, X \backslash Z)$. Then $\tilde{a}=\overline{\tilde{r}}_{\star} \circ T_{\tilde{Z}}(\tilde{a})=\overline{\tilde{r}}_{\star} \circ \tau^{\star}(c)$ and by the first condition in (11), $\tilde{a} \in \operatorname{im} \bar{\chi}$. 
As in the case of de Rham, we see that $H_{\bar{\partial}}^{\bullet \bullet \bullet}(\mathrm{E})$ is a free $H_{\bar{\partial}}^{\bullet \bullet \bullet}(Z)$-module with basis $1, \alpha, \ldots, \alpha^{k-2}, a^{k-1}(Q)$, $\alpha=a^{1}(T)$. Thus we have

$$
H_{\bar{\partial}}^{p-1, q-1}(\mathrm{E}) / \bar{\chi} H_{\bar{\partial}}^{p-k, q-k}(Z) \simeq \bigoplus_{i=0}^{k-2} \alpha^{i} \cdot \tau_{\mathrm{E}}^{\star} H_{\bar{\partial}}^{p-i-1, q-i-1}(Z) \subset H_{\bar{\partial}}^{p-1, q-1}(\mathrm{E}) .
$$

Under the assumption (11), the restriction of the $\bar{\partial}$-Gysin morphism $\left(\bar{i}_{\mathrm{E}}\right)_{\star}=j^{\star} \circ \bar{T}_{\mathrm{E}}: H_{\bar{\partial}}^{p-1 \cdot q-1}(\mathrm{E}) \rightarrow H_{\bar{\partial}}^{p, q}(\tilde{X})$ gives a splitting $\eta$ :

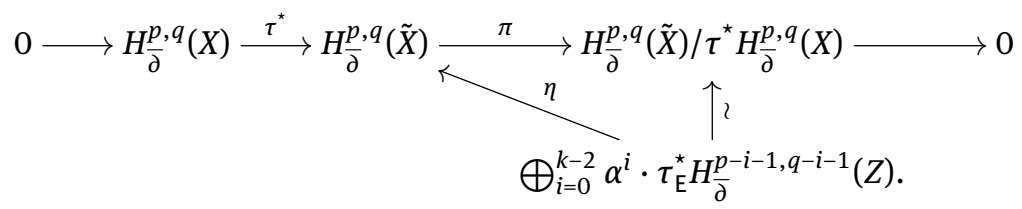

and we have:

Proposition 19. Under the assumption (11), there is an isomorphism

$$
H_{\bar{\partial}}^{p, q}(X) \oplus \bigoplus_{i=0}^{k-2} H_{\bar{\partial}}^{p-i-1, q-i-1}(Z) \stackrel{\sim}{\longrightarrow} H_{\bar{\partial}}^{p, q}(\tilde{X}),
$$

which is given by $\left(x,\left(z_{i}\right)_{i=0}^{k-2}\right) \mapsto \tau^{\star} x+\sum_{i=0}^{k-2}\left(\bar{i}_{\mathrm{E}}\right) \star\left(\alpha^{i} \cdot \tau_{\mathrm{E}}^{\star} z_{i}\right)$ for $x \in H_{\bar{\partial}}^{p, q}(X)$ and $z_{i} \in H_{\bar{\partial}}^{p-i-1, q-i-1}(Z)$.

The theorem follows from Propositions 16 and 19 (cf. Definition 4. 1).

Remark 20. (1) Even if $X$ and $Z$ admit a natural Hodge structure, i.e., satisfy the $\partial \bar{\partial}$-Lemma, it is not clear, from the above arguments, whether or not $\tilde{X}$ has the same property. The problem is that the cohomology of $\mathrm{E}$ contributes to the cohomology of $\tilde{X}$ through the Gysin morphisms and it is not clear if these morphisms send good representatives to good ones as in (H1) and (H2) of Definition 4. 2.

(2) In view of the commutative diagram (15), which is a consequence of Lemma 15, the injectivity of $\tau^{\star}$ on the relative cohomology is equivalent to that of $\chi$. From the definition of $\chi$, we see that this is also equivalent to the injectivity of $\tau_{\mathrm{E}}^{*}$. The injectivity of $\chi$ can also be proven as follows, independently of the injectivity of $\tau^{\star}$.

Recalling that $\tau_{\mathrm{E}}: \mathrm{E}=\mathbb{P}(N) \rightarrow Z$ is a $\mathbb{P}^{k-1}$-bundle, we have the integration along the fibres $\left(\tau_{\mathrm{E}}\right)_{\star}: H_{d R}^{h-2}(\mathrm{E}) \rightarrow H_{d R}^{h-2 k}(Z)$. First we claim that, for $\left(\tau_{\mathrm{E}}\right)_{\star}: H_{d R}^{2 k-2}(\mathrm{E}) \rightarrow H_{d R}^{0}(Z)$, we have

$$
\left(\tau_{\mathrm{E}}\right) \star c^{k-1}(Q)=1 \text {. }
$$

This can be seen from (17), the projection formula and the facts that $\left(\tau_{\mathrm{E}}\right)_{\star} \gamma^{i}=0$, for $i=0, \ldots, k-2$, by dimension reason, and $\left(\tau_{\mathrm{E}}\right)_{*} \gamma^{k-1}=(-1)^{k-1}$, as $\gamma$ restricted to each fibre is the first Chern class of the tautological bundle on $\mathbb{P}^{k-1}$. Then by the projection formula and (21),

$$
\left(\tau_{\mathrm{E}}\right)_{\star} \circ \chi(a)=\left(\tau_{\mathrm{E}}\right)_{\star}\left(c^{k-1}(Q) \smile \tau_{\mathrm{E}}^{\star}(a)\right)=\left(\tau_{\mathrm{E}}\right)_{\star} c^{k-1}(Q) \smile a=a .
$$

Thus the composition $\left(\tau_{\mathrm{E}}\right)_{\star} \circ \chi$ is the identity morphism of $H_{d R}^{h-2 k}(Z)$ so that $\chi$ is injective. Thus $\tau_{\mathrm{E}}^{\star}$ is also injective. If $E$ is Kähler, this follows from [51, Theorem 4.1].

(3) The statement of Proposition 16 is proven in the Kähler context, e.g. in [47, Theorem 7.31] by excision and by the Thom isomorphism in cohomology with $\mathbb{Z}$-coefficients. Presumably, the Kähler condition is necessary there to show that $\chi$ or $\tau_{\mathrm{E}}^{\star}$ is injective using the above-mentioned theorem [51, Theorem 4.1]. The novelty here is the elimination of this restriction by a result of [45] or Lemma 15, which also gives a precise relation between the Thom classes of $Z$ and $E$ and this in turn gives a precise relation between $\tau^{\star}$ and $\chi$.

This subject is treated in the algebraic category in $[21, \S 6.7]$.

(4) In the Dolbeault case, we can show the injectivity of $\bar{\chi}$ similarly as for $\chi$ (cf. (1) above). However this does not directly imply the injectivity of $\tau^{\star}$ on the relative cohomology. The injectivity of $\bar{\chi}$ is equivalent to that of $\tau_{\mathrm{E}}^{\star}: H_{\bar{\partial}}^{p-k, q-k}(Z) \rightarrow H_{\bar{\partial}}^{p-k, q-k}(\mathrm{E})$. If $\mathrm{E}$ is Kähler, the latter again follows from [51, Theorem 4.1]. 
(5) The condition regarding the holomorphically-contractible neighbourhood of $Z$ in Theorem 13 holds, for example, if $Z$ is a point (see Example 21), or if $X$ is a fibration (e.g. a Hopf manifold) with $Z$ a fibre.

(6) The first condition in (11) is implied by the commutativity of the diagram (10), which may be verified for the top degree cohomology using the projection formula.

Example 21 (Blow-up in a point; see also [50, Proposition 3.6]). The very particular case when $Z$ is a point is easier, and follows by the description of the Dolbeault cohomology in [22]. For completeness we outline the proof in this situation. Let $X$ be a compact complex manifold and consider $\tau: \tilde{X} \rightarrow X$ the blow-up of $X$ on a point $p$. If $X$ admits a Hodge structure, then also $\tilde{X}$ does.

We denote by $E=\mathbb{P}^{n-1}=\tau^{-1}(p)$ the exceptional divisor of the blow-up. We recall that the de Rham and Dolbeault cohomologies of $X$ and $\tilde{X}$ are related as follows (see [22, pages 473-474]): for $k \notin\{0,2 n\}$, for $(p, q) \notin\{(0,0),(n, n)\}$,

$$
H^{\bullet}(\tilde{X}, \mathbb{C})=H^{\bullet}(X, \mathbb{C}) \oplus H^{\bullet}(\mathrm{E}, \mathbb{C})
$$

and

$$
H_{\bar{\partial}}^{\bullet \bullet \bullet}(\tilde{X})=H_{\bar{\partial}}^{\bullet \bullet \bullet}(X) \oplus H_{\bar{\partial}}^{\bullet, \bullet}(\mathrm{E}) .
$$

In particular, $h^{p, p}(\tilde{X})=h^{p, p}(X)+1$ and $h^{p, q}(\tilde{X})=h^{p, q}(X)$ for $p \neq q$. Since, by hypothesis, $X$ satisfies the $\partial \bar{\partial}$-lemma and $E$ clearly does, we have that

$$
\begin{aligned}
H^{k}(\tilde{X}, \mathbb{C}) & =H^{k}(X, \mathbb{C}) \oplus H^{k}(\mathrm{E}, \mathbb{C}) \\
& =\bigoplus_{p+q=k} H_{\bar{\partial}}^{p, q}(X) \oplus \bigoplus_{r+s=k} H_{\bar{\partial}}^{r, s}(\mathrm{E}) \\
& =\bigoplus_{t+v=k}\left(H_{\bar{\partial}}^{t, v}(X) \oplus H_{\bar{\partial}}^{t, v}(\mathrm{E})\right)=\bigoplus_{t+v=k} H_{\bar{\partial}}^{t, v}(\tilde{X})
\end{aligned}
$$

and

$$
\begin{aligned}
\overline{H_{\bar{\partial}}^{p, q}(\tilde{X})} & =\overline{H_{\bar{\partial}}^{p, q}(X) \oplus H_{\bar{\partial}}^{p, q}(\mathrm{E})} \\
& =H_{\bar{\partial}}^{q, p}(X) \oplus H_{\bar{\partial}}^{q, p}(\mathrm{E})=H_{\bar{\partial}}^{q, p}(\tilde{X}) .
\end{aligned}
$$

Question 22. We ask whether a submanifold of a manifold satisfying the $\partial \bar{\partial}$-Lemma, still satisfies the $\partial \bar{\partial}$ Lemma. Note that, in general, existence of Hodge structures is not preserved by blow-ups: Claire Voisin suggested to us an example that appears in [48] by Victor Vuletescu: take the blow-up of a Hopf surface inside $\mathbb{S}^{3} \times \mathbb{S}^{3} \times \mathbb{P}^{1}$. (Compare also [50, Concluding Remarks].)

Question 23. We ask whether if $X$ and $Z$ satisfy the $\partial \bar{\partial}-L e m m a$, then we can perform constructions like the deformation to the normal cone for $(X, Z)$ that still satisfies the $\partial \bar{\partial}$-Lemma. We recall that the deformation to the normal cone by MacPherson [21, Chapter 5] allows to modify the pair $(X, Z)$ to the pair $\left(N_{Z \mid X}, Z\right)$ as deformation, where clearly $Z$ has the property of admitting a holomorphically contractible neighbourhood in its normal bundle $N_{Z \mid X}$. We briefly recall the construction, see also [43, Section 8]: consider a 1-dimensional disc $\mathbb{D}$; define $X^{\star}:=\mathrm{Bl}_{Z \times\{0\}}(X \times \mathbb{D}) \backslash \mathrm{Bl}_{Z \times\{0\}}(X \times\{0\})$ that provides a deformation path through $X_{t}^{\star}=X$ to $X_{0}^{\star}=N_{Z \mid X}$. We notice that $N_{Z \mid X}$ is clearly non-compact. We also recall that satisfying the $\partial \bar{\partial}$-Lemma is an open property under deformations [47, Proposition 9.21], but in general it is not closed [5].

Remark 24. If Questions 23 and 22 have positive answers, if we can avoid the technical assumption (11), and if we can prove the naturality of the induced Hodge structures on the blow-up, then our argument would give that the $\partial \bar{\partial}$-Lemma property is defined inside the localization of the category of holomorphic maps with respect to bimeromorphisms, equivalently, modifications. More precisely: Let $f: M \rightarrow N$ be a bimeromorphic map between compact complex manifolds of the same dimension. Then $M$ satisfies the $\partial \bar{\partial}$-Lemma if and only if $N$ does. (The same would be true assuming $M$ Kähler without Question 22.) Indeed, this will follow by the Weak Factorization Theorem for bimeromorphic maps between compact complex manifolds [2, Theorem 0.3.1], [49]. It states that $f$ can be functorially factored as a sequence of blow-ups and blow-downs with non-singular 
centres. For a blow-up $\varphi: V^{\prime} \rightarrow V^{\prime \prime}$, we have that: if $V^{\prime}$ satisfies the $\partial \bar{\partial}$-Lemma, then $V^{\prime \prime}$ does by [16, Theorem 5.22]; if $V^{\prime \prime}$ satisfies the $\partial \bar{\partial}$-Lemma, then $V^{\prime}$ does by Theorem 13. Compare [34, Question 1.2] and [40, Corollary 28].

\section{The orbifold case}

We now consider the orbifold case, applying the Stelzig arguments to the orbifold Dolbeault cohomology studied in [8, 9]. Recall that an orbifold, also called V-manifold [36], is a singular complex space whose singularities are locally isomorphic to quotient singularities $\mathbb{C}^{n} / G$, where $G \subset \mathrm{GL}(n, \mathbb{C})$ is a finite subgroup. Tensors on an orbifold are defined to be locally $G$-invariant. In particular, this yields the notions of orbifold de Rham cohomology and orbifold Dolbeault cohomology, for which we have both a sheaf-theoretic and an analytic interpretation $[8,9,36]$, and Hodge decomposition in cohomology defines the orbifold $\partial \bar{\partial}$-Lemma property.

The following result generalizes the contents of Theorem 1 to orbifolds of global-quotient type, namely, $X / G$, where $X$ is a complex manifold and $G$ is a finite group of biholomorphisms of $X$. We can interpret this case as the smooth case with the further action of a group $G$ : for example, an orbifold morphism $Z / H \rightarrow X /$ $G$ is just an equivariant map $Z \rightarrow X$. The orbifold Dolbeault cohomology of $X / G$ is the cohomology of the complex of $G$-invariant forms, $\left(\left(\wedge^{\bullet}, \bullet X\right)^{G}, \bar{\partial}\right)$. The notion of $\partial \bar{\partial}$-Lemma for orbifolds refers to the cohomological decomposition for the double complex $\left(\left(\wedge^{\bullet \bullet \bullet} X\right)^{G}, \partial, \bar{\partial}\right)$. This result follows directly by the work of Jonas Stelzig and it will let us construct new examples of compact complex manifolds satisfying the $\partial \bar{\partial}$-Lemma, as resolutions of orbifolds obtained starting from compact quotients of solvable Lie groups. (Here, by asking that $j^{o}: Z^{o}=Z / G \rightarrow X^{o}=X / G$ is a suborbifold, we mean that $Z$ is a $G$-invariant submanifold of $X$, and the embedding $j: Z \rightarrow X$ is $G$-equivariant.)

Theorem 25 (see [40]). Let $X^{o}=X / G$ be a compact complex orbifold of complex dimension $n$, and $j^{o}: Z^{o}=$ $Z / G \rightarrow X^{o}$ be a suborbifold of complex dimension $d$ and codimension $k:=n-d$, and consider $\tau^{o}: \tilde{X}_{Z^{o}} \rightarrow X^{o}$ the blow-up of $X^{o}$ along the centre $Z^{o}$. If both $X^{o}$ and $Z^{o}$ satisfy the $\partial \bar{\partial}$-Lemma, then also $\tilde{X}_{Z^{\circ}}$ does satisfy the

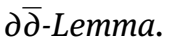

Proof. We first notice that $\tilde{X}_{Z^{\circ}}$ itself is a (possibly smooth) orbifold of global-quotient type. Indeed, by the universal property of blow-up, see e.g. [22, page 604], the action $G \circlearrowleft X$ yields the action $G \circlearrowleft \tilde{X}_{Z}$ the blow-up of $X$ along $Z$. The proof then follows by considering the $E_{1}$-quasi-isomorphism $\wedge^{\bullet \bullet \bullet} \tilde{X}_{Z} \simeq_{1} \wedge^{\bullet, \bullet} X \oplus \bigoplus_{j=1}^{k-1} \wedge^{\bullet-j, \bullet^{-j}} Z$. This means that there is a morphism of double complexes that induces an isomorphism at the first page $E_{1}$ of the Frölicher spectral sequence, that is, the Dolbeault cohomology, see [40, Definition D]. The fact that there is an $E_{1}$-quasi-isomorphism as above is [40, Theorem 23], see also [39]. Since the action of $G$ is compatible with the above morphism, we get also an $E_{1}$-quasi-isomorphism $\left(\wedge^{\bullet \bullet \bullet} \tilde{X}_{Z}\right)^{G} \simeq_{1}\left(\wedge^{\bullet \bullet \bullet} X\right)^{G} \oplus \bigoplus_{j=1}^{k-1}\left(\wedge^{\bullet-j, \bullet-j} Z\right)^{G}$. Recall that the Dolbeault and the de Rham cohomologies of the orbifold are computed as the cohomologies of the complex of $G$-invariant forms, as said above. Therefore the properties of Hodge decomposition for $X$ and $Z$ reflects on the property of Hodge decomposition for $\tilde{X}_{Z}$ by means of the above quasi-isomorphism.

Example 26 (resolution of an orbifold covered by the Iwasawa manifold). In this example, starting from a smooth compact complex manifold which does not satisfy the $\partial \bar{\partial}$-lemma, we construct a simply-connected smooth compact complex manifold that does.

Consider the complex Heisenberg group

$$
G:=\left\{\left(\begin{array}{ccc}
1 & z_{1} & z_{3} \\
0 & 1 & z_{2} \\
0 & 0 & 1
\end{array}\right): z_{1}, z_{2}, z_{3} \in \mathbb{C}\right\} .
$$


It is a nilpotent Lie group, and it is endowed with a bi-invariant complex structure defined by the coframe of $(1,0)$-forms

$$
\varphi^{1}:=d z_{1}, \quad \varphi^{2}:=d z_{2}, \quad \varphi^{3}:=d z_{3}-z_{1} d z_{2} .
$$

They have structure equations

$$
d \varphi^{1}=0, \quad d \varphi^{2}=0, \quad d \varphi^{3}=-\varphi^{1} \wedge \varphi^{2} .
$$

Let $\xi \neq 1$ be a cubic root of the unity, and $\Lambda$ be the lattice generated by 1 and $\xi$. Consider the subgroup $\Gamma$ in $G$ consisting of matrices with entries in $\Lambda$. The compact quotient $M:=G / \Gamma$ is a holomorphically-parallelizable nilmanifold [29]. By [30, 35], the de Rham and Dolbeault cohomologies of $M$ are the same as the cohomologies of the Iwasawa manifold, which are computed for instance in [37].

We consider the following action of the finite group $\mathbb{Z}_{3}$ on $G$ :

$$
\sigma:\left(z_{1}, z_{2}, z_{3}\right) \mapsto\left(\xi z_{1}, \xi z_{2}, \xi^{2} z_{3}\right) .
$$

It is easy to check that the action is linear, and since $\xi^{2}=-1-\xi$ then $\Gamma$ is $\sigma$-invariant. Therefore we get an action on the quotient $M$, and a complex space $M^{o}:=M /\langle\sigma\rangle$ with orbifold singularities. The action on the global co-frame of $(1,0)$-forms becomes

$$
\sigma^{\star}\left(\varphi^{1}\right)=\xi \varphi^{1}, \quad \sigma^{\star}\left(\varphi^{2}\right)=\xi \varphi^{2}, \quad \sigma^{\star}\left(\varphi^{3}\right)=\xi^{2} \varphi^{3} .
$$

We compute the orbifold de Rham and Dolbeault cohomologies by taking the $\sigma$-invariant forms. We have

$$
\begin{aligned}
\wedge^{\bullet} M^{o} & =\left(\wedge^{\bullet} M\right)^{\langle\sigma\rangle} \\
& =\wedge\left\langle 1, \varphi^{13}, \varphi^{23}, \varphi^{1 \overline{1}}, \varphi^{1 \overline{2}}, \varphi^{2 \overline{1}}, \varphi^{2 \overline{2}}, \varphi^{3 \overline{3}}, \varphi^{\overline{1} \overline{3}}, \varphi^{\overline{2} \overline{3}}, \varphi^{12 \overline{3}}, \varphi^{3 \overline{1} \overline{2}}\right\rangle
\end{aligned}
$$

as an algebra, with the only non-trivial differentials

$$
d \varphi^{3 \overline{3}}=\varphi^{12 \overline{3}}-\varphi^{3 \overline{1} \overline{2}}, \quad d \varphi^{12 \overline{3}}=d \varphi^{3 \overline{1} \overline{2}}=\varphi^{12 \overline{1} \overline{2}} .
$$

It is straightforward to check that this complex satisfies the $\partial \bar{\partial}$-Lemma, that is, the orbifold $M^{o}$ satisfies the дð-lemma.

Now we resolve the singularities of $M^{o}$ in order to obtain a simply-connected smooth compact complex manifold satisfying the $\partial \bar{\partial}$-lemma. The procedure is similar to the one described in [17]. The singular locus of $M^{o}$ consists of $3^{3}$ isolated singular points. By blowing-up each point, we get an exceptional divisor $\mathbb{C P}^{2} / \mathbb{Z}_{3}$, where the action is given by

$$
\sigma:\left[z_{1}: z_{2}: z_{3}\right] \mapsto\left[z_{1}: z_{2}: \xi z_{3}\right] .
$$

The singular locus consists now of the isolated point $q:=[0: 0: 1]$ and of the complex projective line $L:=\left\{\left[z_{1}: z_{2}: 0\right]\right\} \subset \mathbb{C P}^{2}$, which both admit a holomorphically contractible neighbourhood. Finally, by blowing-up the $q$ 's and the $L$ 's, we get a smooth model $\tilde{M}$. Thanks to Theorem 25 , the performed operations mantain the $\partial \bar{\partial}$-Lemma property.

In fact, the same argument as [17, Proposition 2.3] adapted to our manifold $M$, which is a principal 2-torus bundle over a 4-torus, yields that $\tilde{M}$ is simply-connected. Moreover, the metric

$$
\omega:=\frac{\sqrt{-1}}{2} \sum_{j=1}^{3} \varphi^{j} \wedge \bar{\varphi}^{j}
$$

on $M$ is $\sigma$-invariant and so it descends to the orbifold $M^{o}$. We can also obtain $\tilde{M}$ by blowing-up $M$ and then by quotienting by $\mathbb{Z}_{3}$. Therefore, $\omega$ yields a balanced metric on $\tilde{M}$ thanks to [3].

Finally, we notice that $\tilde{M}$ is not in class $\mathcal{C}$ of Fujiki, since $M$ is not.

Summarizing the contents of the last example:

Theorem 27. There exists a simply-connected compact complex non-Kähler manifold $\tilde{M}$ such that: it is nonKähler, in fact it does not belong to class $\mathcal{C}$ of Fujiki; it satisfies the d $\bar{\partial}$-Lemma; and it is endowed with a balanced metric. 
Acknowledgement: The authors warmly thank Giovanni Bazzoni, Carlo Collari, Hisashi Kasuya, Sheng Rao, Jonas Stelzig, Valentino Tosatti, Claire Voisin, Victor Vuletescu, Song Yang, Xiangdong Yang, Weiyi Zhang for interesting and useful discussions on many occasions. Thanks also to the anonymous Referees for some suggestions and comments that improve the presentation of the paper. Parts of this work have been written during the visit of the first-named author at the Hokkaido University and that of the second-named author at Università di Firenze: they would like to thank the both Departments for the warm hospitality.

During the preparation of this work, the first-named author has been supported by the Project SIR2014 "Analytic aspects in complex and hypercomplex geometry" (code RBSI14DYEB), by the Projects PRIN "Varietà reali e complesse: geometria, topologia e analisi armonica' and PRIN2017 "Real and Complex Manifolds: Topology, Geometry and holomorphic dynamics" (code 2017JZ2SW5), and by GNSAGA of INdAM. The secondnamed author is supported by the JSPS grant no. 16K05116. The third-named author is supported by Project PRIN “Varietà reali e complesse: geometria, topologia e analisi armonica”, by SIR2014 project RBSI14DYEB "Analytic aspects in complex and hypercomplex geometry", and by GNSAGA of INdAM. The fourth-named author is supported by Project PRIN "Varietà reali e complesse: geometria, topologia e analisi armonica" the Project PRIN 2017 "Real and Complex Manifolds: Topology, Geometry and holomorphic dynamics" and by GNSAGA of INdAM

\section{References}

[1] M. Abate, F. Bracci, T. Suwa, F. Tovena, Localization of Atiyah classes, Rev. Mat. Iberoam. 29 (2013), 547-578.

[2] D. Abramovich, K. Karu, K. Matsuki, J. Wtodarczyk, Torification and factorization of birational maps, J. Amer. Math. Soc. 15 (2002), no. 3, 531-572.

[3] L. Alessandrini, G. Bassanelli, The class of compact balanced manifolds is invariant under modifications, Complex analysis and geometry (Trento, 1993), 1-17, Lecture Notes in Pure and Appl. Math., 173, Dekker, New York, 1996.

[4] D. Angella, H. Kasuya, Bott-Chern cohomology of solvmanifolds, Ann. Global Anal. Geom. 52 (2017), no. 4, 363-411.

[5] D. Angella, H. Kasuya, Cohomologies of deformations of solvmanifolds and closedness of some properties, North-West. Eur. J. Math. 3 (2017), 75-105.

[6] D. Angella, N. Tardini, Quantitative and qualitative cohomological properties for non-Kähler manifolds. Proc. Amer. Math. Soc. 145 (2017) no. 1, 273-285.

[7] D. Angella, A. Tomassini, On the $\partial \bar{\partial}$-Lemma and Bott-Chern cohomology, Invent. Math. 192 (2013), no. 1, 71-81.

[8] W. L. Baily, On the quotient of an analytic manifold by a group of analytic homeomorphisms, Proc. Nat. Acad. Sci. U. S. A. 40 (1954), no. 9, 804-808.

[9] W. L. Baily, The decomposition theorem for $V$-manifolds, Amer. J. Math. 78 (1956), no. 4, 862-888.

[10] G. Bazzoni, M. Fernández, V. Muñoz, A 6-dimensional simply connected complex and symplectic manifold with no Kähler metric, J. Symplectic Geom. 16 (2018), no. 4, 1001-1020.

[11] A. Blanchard, Sur les variétés analytiques complexes, Ann. Sci. l'É.N.S. 73 (1956), no 2, 157-202.

[12] N. Buchdahl, On compact Kähler surfaces, Ann. Inst. Fourier (Grenoble) 49 (1999), no. 1, vii, xi, 287-302.

[13] F. Campana, The class $\mathcal{C}$ is not stable by small deformations, Math. Ann. 290 (1991), no. 1, 19-30.

[14] M. Ceballos, A. Otal, L. Ugarte, R. Villacampa, Invariant complex structures on 6-nilmanifolds: classification, Frölicher spectral sequence and special Hermitian metrics, J. Geom. Anal. 26 (2016), no. 1, 252-286.

[15] L.A. Cordero, M. Fernández, A. Gray and L. Ugarte, Compact nilmanifolds with nilpotent complex structures: Dolbeault cohomology, Trans. Amer. Math. Soc. 352 (2000), no. 12, 5405-5433.

[16] P. Deligne, Ph. A. Griffiths, J. Morgan, D. P. Sullivan, Real homotopy theory of Kähler manifolds, Invent. Math. 29 (1975), no. 3, 245-274.

[17] M. Fernández, V. Muñoz, An 8-dimensional nonformal, simply connected, symplectic manifold, Ann. of Math. (2) 167 (2008), no. 3, 1045-1054.

[18] R. Friedman, On threefolds with trivial canonical bundle, Complex geometry and Lie theory (Sundance, UT, 1989), 103-134, Proc. Sympos. Pure Math., 53, Amer. Math. Soc., Providence, RI, 1991.

[19] R. Friedman, The $\partial \bar{\partial}$-lemma for general Clemens manifolds, Pure Appl. Math. Q. 15 (2019), no. 4, 1001-1028.

[20] A. Frölicher, Relations between the cohomology groups of Dolbeault and topological invariants, Proc. Nat. Acad. Sci. U.S.A. 41 (1955), 641-644.

[21] W. Fulton, Intersection theory, Second edition, Ergebnisse der Mathematik und ihrer Grenzgebiete, 3, Folge, A Series of Modern Surveys in Mathematics, 2, Springer-Verlag, Berlin, 1998. 
[22] Ph. Griffiths, J. Harris, Principles of algebraic geometry, Reprint of the 1978 original, Wiley Classics Library, John Wiley \& Sons, Inc., New York, 1994.

[23] K. Hasegawa, Minimal models of nilmanifolds, Proc. Am. Math. Soc. 106 (1989), no. 1, 65-71.

[24] H. Hironaka, An example of a non-Kählerian complex-analytic deformation of Kählerian complex structures, Ann. Math. (2) 75 (1962), no. 1, 190-208.

[25] N. Honda, T. Izawa, T. Suwa, Sato hyperfunctions via relative Dolbeault cohomology, arXiv: 1807.01831 [math. CV], 2018

[26] H. Kasuya, Hodge symmetry and decomposition on non-Kähler solvmanifolds, J. Geom. Phys. 76 (2014), 61-65.

[27] A. Lamari, Courants kählériens et surfaces compactes, Ann. Inst. Fourier (Grenoble) 49 (1999), no. 1, vii, x, $263-285$.

[28] C. LeBrun, Y.S. Poon, Twistors, Kähler manifolds, and bimeromorphic geometry. II, J. Am. Math. Soc. 5 (1992), no. $2,317-325$.

[29] I. Nakamura, Complex parallelisable manifolds and their small deformations, J. Differential Geometry 10 (1975), 85-112.

[30] K. Nomizu, On the cohomology of compact homogeneous spaces of nilpotent Lie groups, Ann. of Math. (2) 59 (1954), no. 3 , 531-538.

[31] D. Popovici, Stability of strongly Gauduchon manifolds under modifications, J. Geom. Anal. 23 (2013), no. 2, 653-659.

[32] D. Popovici, Aeppli cohomology classes associated with Gauduchon metrics on compact complex manifolds, Bull. Soc. Math. France 143 (2015), no. 4, 763-800.

[33] D. Popovici, Volume and self-intersection of differences of two nef classes, Ann. Sc. Norm. Super. Pisa Cl. Sci. (5) 17 (2017), no. 4, 1255-1299.

[34] S. Rao, S. Yang, X. Yang, Dolbeault cohomologies of blowing up complex manifolds, J. Math. Pures Appl. (9) 130 (2019), 68-92.

[35] Y. Sakane, On compact complex parallelisable solvmanifolds, Osaka J. Math. 13 (1976), no. 1, 187-212.

[36] I. Satake, On a generalization of the notion of manifold, Proc. Nat. Acad. Sci. U.S.A. 42 (1956), no. 6, 359-363.

[37] M. Schweitzer, Autour de la cohomologie de Bott-Chern, Prépublication de l'Institut Fourier no. 703 (2007), arXiv:0709.3528.

[38] J. Stelzig, Double Complexes and Mixed Hodge Structures as Vector Bundles, PhD thesis, WWU, Münster, 2018.

[39] J. Stelzig, The Double Complex of a Blow-up, to appear in Internat. Math. Res. Not. IMRN, DoI: 10.1093/imrn/rnz139, arXiv: 1808.02882.

[40] J. Stelzig, On the Structure of Double Complexes, arXiv: 1812.00865.

[41] T. Suwa, Indices of vector fields and residues of singular holomorphic foliations, Actualités Mathématiques, Hermann, Paris, 1998.

[42] T. Suwa, Residue theoretical approach to intersection theory, Contemp. Math. Amer. Math. Soc. 459 (2008), $207-261$.

[43] T. Suwa, Čech-Dolbeault cohomology and the $\bar{\partial}$-Thom class, Singularities-Niigata-Toyama 2007, 321-340, Adv. Stud. Pure Math., 56, Math. Soc. Japan, Tokyo, 2009.

[44] T. Suwa, Relative Dolbeault cohomology, arXiv:1903.04710.

[45] N. Tardini, Relative Čech-Dolbeault homology and applications, Ann. Mat. Pura Appl., 199 (2020), no. 3, 985-995.

[46] V. Tosatti, B. Weinkove, Hermitian metrics, $(n-1, n-1)$ forms and Monge-Ampère equations, J. Reine Angew. Math. 755 (2019), 67-101

[47] C. Voisin, Hodge theory and complex algebraic geometry. I, Translated from the French by Leila Schneps, Reprint of the 2002 English edition, Cambridge Studies in Advanced Mathematics, 76, Cambridge University Press, Cambridge, 2007.

[48] V. Vuletescu, Exemples de faisceaux cohérents sans résolution localement libre en dimension 3, C. R. Math. Acad. Sci. Paris 350 (2012), no. 7-8, 411-412.

[49] J. Wtodarczyk, Toroidal varieties and the weak factorization theorem, Invent. Math. 154 (2003), no. 2, 223-331.

[50] S. Yang, X. Yang, Bott-Chern blow-up formula and bimeromorphic invariance of the $\partial \bar{\partial}$-Lemma for threefolds, arXiv: 1712.08901.

[51] R. O. Wells, Comparison of de Rham and Dolbeault cohomology for proper surjective mappings, Pacific J. Math. 53 (1974), 281-300.

[52] C.-C. Wu, On the geometry of superstrings with torsion, Thesis (Ph.D.)Harvard University, Proquest LLC, Ann Arbor, MI, 2006. 